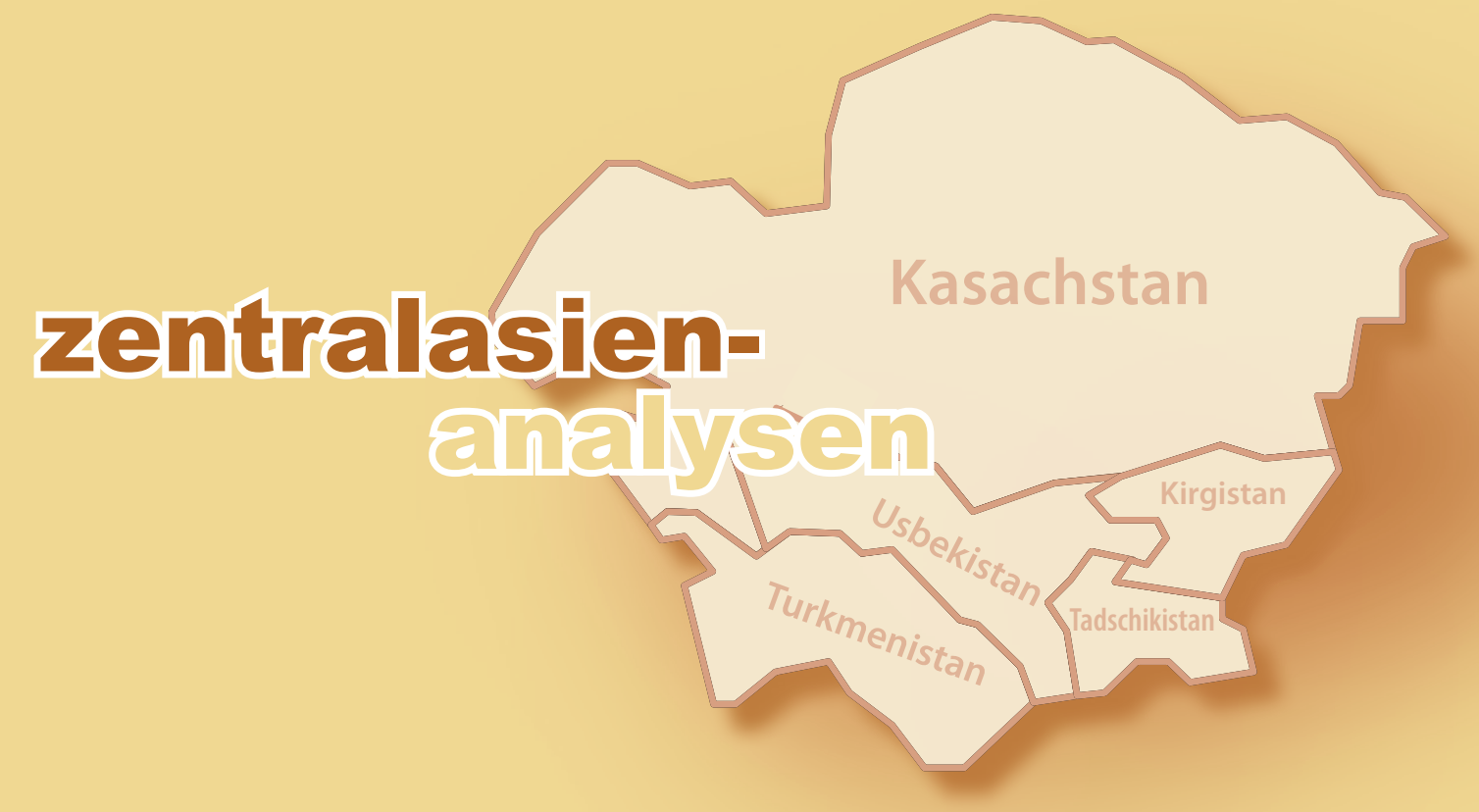

\title{
REGIONALE ZUSAMMENARBEIT IN ZENTRALASIEN
}

\section{ANALYSE}

Ein Ende der »Kaltzeit»?

Positive Tendenzen in den Beziehungen zwischen den Staaten Zentralasiens

Von Raichan Taschtemchanowa und Dschanar Medeubajewa, Astana

\section{TABELLEN ZUM TEXT}

Importe der zentralasiatischen Staaten untereinander

Überregional bedeutende Grenzübergänge zwischen den Staaten Zentralasiens

\section{CHRONIK}

24. Juni - 21. Juli $2017 \quad 8$

Kasachstan $\quad 8$

$\begin{array}{lr}\text { Kirgistan } & 10\end{array}$

$\begin{array}{ll}\text { Tadschikistan } & 12\end{array}$

Turkmenistan 14

$\begin{array}{ll}\text { Usbekistan } & 15\end{array}$ 


\section{Ein Ende der »Kaltzeit«?}

\section{Positive Tendenzen in den Beziehungen zwischen den Staaten Zentralasiens}

Von Raichan Taschtemchanowa und Dschanar Medeubajewa, Astana

\section{Zusammenfassung}

Die Beziehungen zwischen den fünf zentralasiatischen Staaten waren seit ihrer Unabhängigkeit schwach entwickelt und durch ungelöste Konflikte geprägt, die langfristig die innere wie äußere Stabilität der Länder gefährdeten - eine Phase, die von den Autorinnen dieses Beitrags als Kaltzeit charakterisiert wird. Die weltpolitische Lage hat schon in den letzten Jahren zu einer allerersten Annäherung, einer leichten Erwärmung, geführt. Die neuen außenpolitischen Prioritäten des Nachfolgers des langjährigen usbekischen Präsidenten Islam Karimow, Schawkat Mirsijojew, lassen das Eis jetzt noch stärker schmelzen. Es besteht die Hoffnung, dass es sich nicht nur um ein kurzfristiges Tauwetter handelt, sondern eine längerfristige Warmzeit.

\section{Kaltzeit in Zentralasien und die Folgen}

Die Republiken Kasachstan, Usbekistan, Turkmenistan, Kirgistan und Tadschikistan sind bis auf den heutigen Tag Geiseln ihrer sowjetischen Vergangenheit und durch ein ganzes Bündel ungelöster zwischenstaatlicher Probleme miteinander verbunden. Die Tatsache, dass diese Länder in ihrem Bestreben, Teil der internationalen Gemeinschaft zu werden, in der Lage waren, mit zahlreichen Staaten der Welt Beziehungen aufzubauen, während gleichzeitig ihre Kontakte zu den unmittelbaren Nachbarn unbefriedigend blieben, ist paradox. Von außen betrachtet sieht es so aus, als ob sich die zentralasiatischen Staaten untereinander einfach ignorieren. Schon ein flüchtiger Blick auf die zwischenstaatlichen Beziehungen seit der Unabhängigkeit zeigt, dass zwischen ihnen Misstrauen, Argwohn und Feindseligkeit herrschen. Diese Kälte, welche die Region gefangen hält, wurde fast nie von einer Erwärmung oder einem Tauwetter unterbrochen. Dafür sind sowjetische Erblasten in Territorialfragen verantwortlich, die zusätzlich durch aktuelle Entwicklungen belastet werden.

Die Lage wird dadurch erschwert, dass die Grenzverläufe zwischen den zentralasiatischen Staaten, im Gegensatz zu den Außengrenzen der Gesamtregion, bis heute nicht vollständig geklärt sind. Von den fünf Republiken Zentralasiens hat nur Turkmenistan seine gesamte Landgrenze durch entsprechende Abkommen mit Usbekistan und Kasachstan delimitiert. Kasachstan hat die wichtigsten Grenzfragen mit Usbekistan und Kirgistan geregelt. Doch die Grenzstreitigkeiten zwischen Usbekistan, Tadschikistan und Kirgistan sind bis heute nicht beigelegt. Außerdem sitzen genau diese drei Staaten im Ferganatal sozusagen in der Falle, denn alle haben Exklaven auf den Territorien ihrer Nachbarstaaten. Seit der Unabhängigkeit hat es an den Grenzen ständig Zwischenfälle (sogar mit tödlichem Ausgang) wegen des Zugangs zu Wasser, um Weidegründe oder als Ausdruck des Protests gegen die strengen Regelungen des
Grenzübertritts gegeben. Das führte immer wieder zu Waffengebrauch bei Grenzschützern, und anschließend beschuldigten sich die Staaten gegenseitig der Aggression. So schickte beispielsweise Usbekistan 2010 während eines solchen Konflikts in seiner in Kirgistan gelegenen Exklave Soch Luftlandetruppen und Panzer an die Grenze. Für alle drei Staaten liegt der Gedanke nahe, dass der unmittelbare Anschluss ihrer Exklaven an das Hauptland auch andere Probleme lösen würde - zum Beispiel den Zugang zu Wasserressourcen oder wichtigen Transportwegen. Es ist bereits mehrfach zu Konflikten zwischen den Anliegerstaaten der Oberläufe der großen zentralasiatischen Flüsse (Kirgistan und Tadschikistan) und den Unterliegern (Kasachstan, Usbekistan und Turkmenistan) gekommen. Im Winter 2000 beispielsweise stellte Usbekistan seine Gaslieferungen an Kirgistan ein, während die kirgisische Seite zur Stromgewinnung Wasser aus dem Toktogul-Stausee abließ das zu diesem Zeitpunkt aber nicht zur Bewässerung der usbekischen Baumwollfelder benötigt wurde, sondern Überschwemmungen verursachte. Als Antwort darauf zog Usbekistan an der Grenze Truppen zusammen, und der Konflikt wäre beinahe eskaliert, wurde aber zum Glück gelöst. Das Beispiel zeigt dennoch, dass die Bemerkung des damaligen usbekischen Präsidenten Islam Karimow, der Bau von Wasserkraftwerken in Tadschikistan und Kirgistan könne »nicht nur zu einem Zusammenstoß, sondern zu einem Krieg« führen, nicht nur eine Worthülse war.

Die Unfähigkeit der politischen Eliten Zentralasiens, das zwischenstaatliche Wassermanagement der Region in den Griff zu bekommen, hat die Aufmerksamkeit der internationalen Gemeinschaft geweckt. So war beispielsweise Berlin im Jahre 2015 Schauplatz von Diskussionen über die Wasserfrage in Zentralasien. Der Titel der internationalen Konferenz, "Wasser und gutnachbarschaftliche Beziehungen in Zentralasien", spricht für sich. Doch die offiziellen Treffen der Staatsoberhäup- 
ter, Forschungsarbeiten von Experten und zahlreichen Empfehlungen internationaler Organisationen haben nicht zu einer Verbesserung der Situation im Bereich der Wasser- und Hydroenergieressourcen geführt. Es ist kein Geheimnis, dass die nationalen Gesetzgebungen in den betreffenden Ländern in dieser Frage recht einseitig nur die Interessen des eigenen Staates vertreten. Die Regierungen begreifen nicht, dass die mangelnde Koordinierung der Politik in diesem Bereich in einigen Ländern Zentralasiens zu höchst unliebsamen und gravierenden sozioökonomischen und ökologischen und letztlich destabilisierenden Folgeproblemen führen kann.

Die schwach entwickelten zwischenstaatlichen Beziehungen trugen zur weiteren Konservierung verschleppter grenzüberschreitender sozioökonomischer Probleme bei, während gleichzeitig schon ganz neue, globale Herausforderungen aufkamen. Die weltweit gesunkenen Preise für Öl, Gas sowie Metalle, welche die Exportgrundlage der zentralasiatischen Staaten bilden, nehmen ihnen alle Chancen auf sozioökonomische Verbesserungen.

Ein Beispiel für die fehlende staatliche Steuerungskapazität ist die massive Abwertung der nationalen Währungen im Jahr 2015. Der Wertverlust schwankte zwischen $25 \%$ und $85 \%$. Die Abwertung war ein schwerer Schlag für das wirtschaftliche und soziale Leben, vor allem in Kirgistan und Tadschikistan, die von Warenimporten abhängig sind. Auch der Wertverfall des russischen Rubels drückt auf die Kurse der zentralasiatischen Währungen. Die Region ist aufgrund ihrer eng mit der Russländischen Föderation verflochtenen Wirtschafts- und Finanzsysteme auch negativ von den Folgen der westlichen Sanktionen gegen Russland betroffen. Kirgistan, Tadschikistan und Usbekistan haben zudem unter den sinkenden Rücküberweisungen ihrer nach Russland ausgereisten Arbeitsmigranten gelitten.

Das außerordentlich hohe Bevölkerungswachstum in Usbekistan und Tadschikistan wird noch für lange Zeit eine Herausforderung für die soziale und wirtschaftliche Sicherheit darstellen. Besonders hervorzuheben ist, dass $70 \%$ der Bevölkerung Usbekistans unter 25 Jahre alt sind. Zwar sind die Kinderzahlen pro Familie (auf eine mittlere Größe) gefallen, doch die Zuwachsraten aufgrund der sehr jungen Bevölkerung (im heiratsfähigen Alter) insgesamt weiterhin hoch. Diese Tatsache verbunden mit den ungelösten sozioökonomischen Problemen können die Region mittelfristig in einen Strudel der Instabilität reißen. Und von dort ist es nicht weit bis zu jenem Grad der Islamisierung, bei dem ein zweiter "Balkan" und ein zweites Afghanistan ein gefährliches "zentralasiatisches« Konglomerat bilden. Angesichts solcher Bedrohungen könnte die Vertiefung zwischenstaat- licher Beziehungen und eine enge Kooperation zwischen den Staaten der Region die Destabilisierung Zentralasiens abwenden.

\section{Temperaturanstieg dank äußerer Herausforderungen}

Nach Ansicht vieler zentralasiatischer Experten ist in den letzten Jahren eine gewisse Erwärmung in den Beziehungen zwischen den zentralasiatischen Staaten zu beobachten. Es liegt nahe, diesen Trend in Beziehung zum Tod des usbekischen Präsidenten Islam Karimow (im September 2016) zu setzen, der mit seiner Ablehnung einer regionalen Zusammenarbeit den gedämpften Ton der innerregionalen Diplomatie vorgab. Der von dem jetzigen Präsidenten Usbekistans, Schawkat Mirsijojew, eingeleitete politische Neustart in den Beziehungen mit den Nachbarn zeigt, dass die Karimow-Epoche der Feindschaft mit Turkmenistan, Kasachstan, Kirgistan und Tadschikistan der Vergangenheit angehört. Die Gesamtsituation wäre allerdings wesentlich unkomplizierter, wenn alles einzig vom Verhalten Usbekistans abhinge.

Einige Anzeichen für eine Klimaänderung in Zentralasien waren auch schon vor dem Tod Karimows zu beobachten. Die Gründe für die Erwärmung der zwischenstaatlichen Beziehungen muss man in grundsätzlicheren Aspekten der regionalen Entwicklung suchen. Die Betrachtung Zentralasiens als Randzone der Weltpolitik erfährt heute im Zusammenhang mit globalen geopolitischen Spannungen eine Veränderung. Es wäre aber kurzsichtig, Zentralasien nur im Schatten der Rivalität der großen »Player« der Weltpolitik zu sehen. Auch die Begriffe "natürliche Ressourcen", "Transportkorridore" und "Pipelines" verleiten zu einer allzu simplen Sicht auf Zentralasien.

Der beste Weg zum Verständnis der aktuellen Lage der zentralasiatischen Republiken ist eine sorgfältige und pragmatische Analyse ihrer Entwicklung von innen heraus. Befürchtungen, dass sich Zentralasien in eine Zone dauerhafter Instabilität und Quelle diverser Bedrohungen verwandeln könnte, haben sich nicht bewahrheitet. Auch eine Islamisierung in der von einigen Experten prognostizierten, radikalen Variante ist nicht zu beobachten. Tatsächlich vollzieht sich ein langanhaltender Prozess der Herausbildung von Nationalstaaten. Es hat eine Elitenkonsolidierung stattgefunden, zwischen den sozialen und politischen Hauptgruppen wurde ein Konsens erreicht (in einigen Fällen mit nicht immer zivilisierten Methoden) und es wurden die Grundlagen für ein Wirtschaftssystem gelegt, das Elemente des früheren sowjetischen Systems mit Marktbeziehungen kombiniert. Es scheint, dass die Staaten Zentralasiens, nachdem sie den »Freudentaumel der Souveränität« durchlebt haben, nun in der Mitte des zweiten Jahrzehnts des 
21. Jahrhunderts Kurs in Richtung einer nüchternen, pragmatischeren Regionalpolitik genommen haben.

Nach Ansicht einer Reihe von Experten haben auch die Ereignisse in der Ukraine einen Einfluss auf die geopolitische Klimaerwärmung zwischen den Staaten Zentralasiens gehabt. Der Kiewer Majdan 2014, der in eine Katastrophe für die gesamte Ukraine mündete, die Annexion der Krim und die einseitige Proklamierung von Republiken in der Ostukraine haben zu Ernüchterung in Zentralasien geführt. Die deutliche Zunahme diplomatischer Aktivitäten auf regionaler Ebene ist ein Indiz dafür. Ganz unerwartet machte z. B. der kirgisische Präsident Almasbek Atambajew 2014 einen offiziellen Besuch in Turkmenistan, der in einer herzlichen und produktiven Atmosphäre verlief. Noch mehr Aufmerksamkeit rief der Besuch Islam Karimows in Astana im November des gleichen Jahres hervor. Aus den Verlautbarungen Karimows während seiner dortigen Auftritte wurde klar, dass die damaligen weltpolitischen Ereignisse ein Hauptgrund für seinen radikalen Sinneswandel waren. Der usbekische Führer war zu dem Schluss gelangt, dass sein Land angesichts der aktuellen problematischen Lage einen sicheren Rückhalt brauchte. $\mathrm{Zu}$ diesem Zeitpunkt hatte Usbekistan seine Mitgliedschaft in der CSTO (Organisation des Vertrags über kollektive Sicherheit) ausgesetzt, die amerikanische Militärbasis in Termes geschlossen und es befand sich in Konflikten mit Tadschikistan und Kirgistan um Wasserressourcen. Im Fall einer militärisch-politischen oder terroristischen Bedrohung hätte das Land weder bei Russland, noch bei den USA einschließlich der EU Unterstützung gefunden. In dieser Situation erinnerte sich Karimow an seine direkten Nachbarn und auch daran, dass zwischen Kasachstan und Usbekistan bereits ein Jahr zuvor ein Vertrag über strategische Partnerschaft unterzeichnet worden war. Wenn man das wortkarge Verhalten des usbekischen Führers bei früheren Besuchen Kasachstans mit seinem Auftreten Ende 2014 vergleicht, dann kann man sich voll und ganz der These von einer ganz neuen Etappe in den kasachisch-usbekischen Beziehungen noch unter Karimow anschließen. 2014 gab es ein weiteres Aufsehen erregendes Ereignis: Zum vierten Gipfeltreffen der Führer der Turkstaaten im türkischen Bodrum reiste erstmals der Präsident Turkmenistans, Gurbanguly Berdymuchamedow, an. Dabei ist im vorliegenden Kontext weniger von Bedeutung, dass das offiziell neutrale Land sich für ein Forum turksprachiger Länder öffnete, als dass sich darin ein neuer Trend zu wechselseitigen Beziehungen Turkmenistans mit seinen Nachbarn sowohl bilateral als auch im Rahmen multilateraler Foren zeigte.

Zweifellos haben auch die alle fünf Staaten betreffenden Bedrohungen aus dem Süden, insbesondere Afgha- nistan, und die Gefahren, die von aus Syrien zurückkehrenden zentralasiatischen Kämpfern ausgehen, einen Einfluss auf den Annäherungsprozess.

\section{Ende der Kaltzeit nach Karimows Tod}

Natürlich wird der Neustart der Beziehungen zwischen den zentralasiatischen Republiken auch direkt mit dem Machtantritt des neuen Präsidenten Usbekistans, Schawkat Mirsijojew, verknüpft. Seit seiner Amtsübernahme hat das Land Kurs auf Erneuerung und Reformen in allen Schlüsselbereichen des gesellschaftlichen und politischen Lebens genommen, auch wenn manche Experten vor der Illusion warnen, dass Mirsijojew eine revolutionäre Politik verfolgen wird. Nach ihrer Ansicht steht seine Politik in der Kontinuität seines Vorgängers. Wie der stellvertretende Außenminister Dschawlon Wachabow im Januar 2017 erklärte, sollen die Beziehungen mit den Nachbarstaaten nun Priorität in der Außenpolitik Usbekistans haben.

Als Beleg für die Aufrichtigkeit der Absichtserklärungen der neuen usbekischen Führung kann man den neuen Kurs bei den Verhandlungen zur Delimitierung und Demarkation der mit den Nachbarn strittigen Grenzen anführen. Seit Ende 2016 unternimmt Taschkent konkrete Schritte, um die Konflikte an der Grenze zu Kirgistan zu entschärfen. Parallel dazu werden Gespräche mit Kasachstan und Tadschikistan über unklare Grenzabschnitte geführt. Die usbekische Initiative hat bei den Nachbarn Unterstützung gefunden. Man darf jedoch keine sofortige Lösung von Problemen erwarten, die sich über Jahrzehnte angehäuft und das Leben mehrerer Generationen vergiftet haben. Doch besonders die neue Intensität und Dynamik der usbekisch-kirgisischen Beziehungen, die früher äußerst angespannt und oft konfrontativ waren, geben zu Hoffnungen Anlass. Die Erfolge, die bisher hinsichtlich der vertraglichen Ausgestaltung der Grenzziehung zwischen Usbekistan und Kirgistan erreicht wurden (49 Abschnitte wurden festgelegt), sind Indiz für das ernsthafte Engagement beider Seiten.

Auch die positiven Entwicklungen in den usbekischtadschikischen Beziehungen machen Hoffnung auf eine langfristige Stabilisierung der Region. Wenn man sich daran erinnert, dass die Konflikte zwischen diesen beiden Staaten ein solches Ausmaß erreicht hatten, dass es zu einem »Eisenbahnkrieg" kam, zur Sprengung einer Brücke auf usbekischem Territorium, die Tadschikistan mit anderen GUS-Staaten verband, und zur Verminung einer neutralen Zone an der usbekisch-tadschikischen Grenze, dann wird verständlich, wie wichtig und vielversprechend die jüngsten Versuche zur Verbesserung der Lage sind. Im vergangenen Jahr wurde der Eisenbahnverkehr zwischen Usbekistan und Tadschikistan 
neu geregelt und einige für beide Seiten problematische Grenzkontrollen abgeschafft. Auch die Flugverbindung zwischen den Hauptstädten wurde wieder in Betrieb genommen.

Das herausragende Ereignis war jedoch der Baubeginn des Wasserkraftwerks Rogun in Tadschikistan im Oktober 2016 bzw., genauer gesagt, nicht der Start der Bauarbeiten als solcher, sondern die Reaktion Usbekistans darauf. Noch vor ungefähr einem Jahr, im Juni 2016, als Karimow noch am Leben war, schickte der heutige Präsident Mirsijojew in seiner damaligen Eigenschaft als Premierminister einen Drohbrief an die tadschikische Regierung, in dem ernsthafte Konsequenzen im Falle eines Beginns der Bautätigkeit angedroht wurden. Als nun jedoch der tadschikische Präsident Emomali Rachmon im Oktober 2016 mit einem Bulldozer einen Erdwall zusammenschob und so symbolisch den Startschuss für die Errichtung des Kraftwerks gab, war von usbekischer Seite kein Sterbenswörtchen zu hören. Unmittelbar danach empfing der usbekische Außenminister Abdulasis Kamilow den stellvertretenden tadschikischen Minister für Energie und Wasserressourcen, Sultan Rachimsoda. Nach offiziellen Angaben diskutierten die Parteien Fragen der effektiven regionalen Nutzung von Hydroenergie, nach Meinung einer Reihe von Experten versuchten die Tadschiken aber vor allem ihre Kollegen davon zu überzeugen, dass der Bau des Wasserkraftwerks nicht zu Lasten Usbekistans gehen wird. Offensichtlich waren ihre Argumente überzeugend, zumindest kam bis heute keine negative Reaktion aus Taschkent. Für die usbekische Regierung haben jetzt wirtschaftliche Interessen Priorität, beispielsweise die Erschließung des tadschikischen Marktes für die usbekische Autoindustrie.

Auch in den usbekisch-turkmenischen Beziehungen lassen sich positive Entwicklungen beobachten. Im Januar 2017 wurde die gemeinsame Grenze, die vier Jahre zuvor geschlossen worden war, endlich wieder für den visafreien Übertritt (mit einer Aufenthaltsdauer von drei Tagen innerhalb eines Monats) für Bürger der Grenzgebiete beider Staaten geöffnet. Die Initiative dafür ging vom Präsidenten Usbekistans aus, der auch die Grenzkontrollpunkte medienwirksam einweihte. Der Besuch des usbekischen Präsidenten in Turkmenistan im März dieses Jahres hat jedoch alle völlig überrascht. Es handelte sich um den ersten auswärtigen Staatsbesuch Mirsijojews in seiner Eigenschaft als Präsident überhaupt - man hatte allgemein erwartet, dass er ihn in Russland oder Kasachstan absolvie- ren würde. Ergebnis des Treffens der beiden Präsidenten war die Unterzeichnung eines Vertrags über strategische Partnerschaft sowie anderer wichtiger Grundsatzdokumente über Zusammenarbeit in Wirtschafts- und Transportfragen. Mit diesem Besuch hat der usbekische Führer eine gewisse Neutralität und seine Unabhängigkeit von den außenpolitischen Orientierungen anderer "Player« in der Region demonstriert. Aber natürlich ist die Bereinigung der Beziehungen mit Turkmenistan auch durch die Notwendigkeit motiviert, die Transportkorridore auszubauen, die beide Länder mit der Außenwelt verbinden.

\section{Erwärmung oder Tauwetter?}

Handelt es sich um eine langfristige und unumkehrbare Erwärmung des zwischenstaatlichen Klimas in Zentralasien oder nur um ein kurzfristiges Tauwetter? Manche Experten sind bezüglich der Dauerhaftigkeit dieses Prozesses skeptisch: Das Eis des Misstrauens und des Argwohns zwischen den Ländern sei inzwischen allzu dick. Natürlich vollziehen sich die Lösung aller strittigen Fragen und die Harmonisierung der Beziehungen nicht an einem Tag. Sowohl innen- wie außenpolitische Faktoren werden Einfluss auf die weitere Entwicklung der internationalen Prozesse in Zentralasien haben. Für eine Prognose über die weiteren Perspektiven der innerregionalen Diplomatie spielt die Tatsache, dass die politischen Regime in praktisch allen Staaten der Region übermäßig personenzentriert sind, eine große Rolle. Nicht nur Experten, sondern auch zentralasiatische Politiker glauben, dass Sym- oder Antipathien zwischen den Staatsoberhäuptern in starkem Maße die Richtung der Außenpolitik ihrer Länder beeinflussen. Es ist vorstellbar, dass dieser Umstand eine positive Rolle beim Neustart der Beziehungen in Zentralasien gespielt hat und dass die freundschaftliche Stimmung der Staatsoberhäupter auch in Zukunft zum endgültigen Abtauen des Eises des Misstrauens und der Feindseligkeit in der Region beitragen wird. Es gibt Grund zur Hoffnung, dass das Streben nach langfristigen Kontakten mit den Nachbarn zum beiderseitigen Nutzen, Pragmatismus sowie das Gefühl, unter den gegenwärtigen Bedingungen aufeinander angewiesen zu sein, sich gegen eine einzig auf den eigenen nationalen Interessen beruhende Politik durchsetzen.

Aus dem Russischen von Brigitte Heuer

Informationen über die Autorinnen und Lesetipps finden Sie auf der nächsten Seite. 
Über die Autorinnen:

Raichan Taschtemchanowa ist habilitierte Historikerin und Professorin an der Nationalen Eurasischen Gumiljow-Universität in Astana, Kasachstan. Ihr Forschungsinteresse gilt den Beziehungen zwischen Zentralasien und den europäischen Staaten in historischer und aktueller Perspektive.

Dschanar Medeubajewa hat in Geschichtswissenschaften promoviert und ist ebenfalls Professorin an der Nationalen Eurasischen Gumiljow-Universität, Astana. Sie ist Expertin für die Außenpolitik Kasachstans und für Sicherheitsfragen in Zentralasien.

Lesetipps:

- Abulkhairkhan Zhunisbek, Uzbekistan's Foreign Policy under the New President, Politics, Foreign Policy and Security Research Group, Eurasian Research Institute, Akhmet Yassawi University, 14.7.2017, = <http://eurasianresearch.org/en/research/comments/foreign-policy/uzbekistan $\%$ E2\%80\%99s-foreign-policy-under-new-president>

- Bilahari Kausikan, S. Frederick Starr, Yang Cheng, Central Asia: All Together Now, The American Interest, 16.6.2017, = <https://www.the-american-interest.com/2017/06/16/central-asia-all-together-now/>

- Majlis Podcast: New Uzbek President Tries His Hand At Foreign Policy, RFE/RL Qishloq Ovozi, 12.3.2017, = <https://www.rferl.org/a/qishloq-ovozi-majlis-podcast-uzbekistan-mirziyaev-foreign-policy/28364968.html>

\section{Importe der zentralasiatischen Staaten untereinander}

Tabelle 1: Gesamtimporte (Mio. US-Dollar) ...

\begin{tabular}{|c|c|c|c|c|c|}
\hline & 2000 & 2004 & 2008 & 2012 & 2015 \\
\hline \multicolumn{6}{|c|}{... nach Kasachstan aus: } \\
\hline Kirgistan & 30,1 & 91,1 & 171,2 & 333,1 & 200,6 \\
\hline Tadschikistan & 4,4 & 3,5 & 8,7 & 68,2 & 218,4 \\
\hline Turkmenistan & 43,4 & 75,6 & 220,3 & 179 & 214,4 \\
\hline Usbekistan & 70,5 & 227,6 & 516,1 & 817,2 & 587,8 \\
\hline \multicolumn{6}{|c|}{... nach Kirgistan aus: } \\
\hline Kasachstan & 57,4 & 203 & 376,5 & 518,7 & 635,6 \\
\hline Tadschikistan & 1,9 & 2,4 & 3,5 & 4 & 6,4 \\
\hline Turkmenistan & 18,7 & 1,4 & 2,2 & 1,8 & 4,4 \\
\hline Usbekistan & 74,6 & 52 & 160,1 & 63,2 & 70 \\
\hline \multicolumn{6}{|c|}{... nach Tadschikistan aus: } \\
\hline Kasachstan & 82,4 & k. A. & k. A. & k. A. & k. A. \\
\hline Kirgistan & 7,6 & k. A. & k. A. & k. A. & k. A. \\
\hline Turkmenistan & 29,3 & k. A. & k. A. & k. A. & k. A. \\
\hline Usbekistan & 185,2 & k. A. & k. A. & k. A. & k. A. \\
\hline \multicolumn{6}{|c|}{... nach Turkmenistan aus: } \\
\hline Kasachstan & 19,7 & k. A. & k. A. & k. A. & k. A. \\
\hline Kirgistan & 4,6 & k. A. & k. A. & k. A. & k. A. \\
\hline Tadschikistan & 6,7 & k. A. & k. A. & k. A. & k. A. \\
\hline Usbekistan & 35,3 & k. A. & k. A. & k. A. & k. A. \\
\hline
\end{tabular}

Anm.: Für Usbekistan liegen keine Daten vor.

Quelle: UN Comtrade: <https://comtrade.un.org/> 


\section{Überregional bedeutende Grenzübergänge zwischen den Staaten}

\section{Zentralasiens}

Tabelle 1: Status von überregional bedeutenden Grenzübergängen zwischen den Staaten Zentralasiens (Stand 2017)

\begin{tabular}{|l|c|c|c|c|c|}
\hline & offen & zeitweise offen & geschlossen & $\begin{array}{c}\text { bilateral } \\
\text { geöffnet, nur } \\
\text { für Bürger der } \\
\text { anliegenden } \\
\text { Staaten } \\
\text { geöffnet }\end{array}$ & $\begin{array}{c}\text { keine Angaben } \\
\text { vorhanden }\end{array}$ \\
\hline Kasachstan/Kirgistan & 4 & 1 & 1 & 2 & 1 \\
\hline Kasachstan/Turkmenistan & 1 & & & & \\
\hline Kasachstan/Usbekistan & 3 & 1 & & & \\
\hline Kirgistan/Tadschikistan & 3 & & & & \\
\hline Kirgistan/Usbekistan & 1 & & 2 & & 2 \\
\hline Tadschikistan/Usbekistan & 2 & & & & 1 \\
\hline Usbekistan/Turkmenistan & 3 & & & & \\
\hline
\end{tabular}

Quelle: Caravanistan; <http://caravanistan.com/border-crossings/>

\section{Exklaven in Zentralasien}

Tabelle 1: Exklaven in Zentralasien

\begin{tabular}{|c|c|c|c|c|}
\hline Name & $\begin{array}{c}\text { Staatliche } \\
\text { Zugehörigkeit }\end{array}$ & $\begin{array}{l}\text { Territoriale Lage } \\
\text { in ... }\end{array}$ & Bevölkerung & $\begin{array}{c}\text { Ethnische } \\
\text { Zusammensetzung }\end{array}$ \\
\hline Barak & Kirgistan & Usbekistan & 600 & $100 \%$ kirgisisch \\
\hline Chonkara & Usbekistan & Kirgistan & 0 & [Weideland] \\
\hline Dschangail & Usbekistan & Kirgistan & 0 & [Weideland] \\
\hline Sarwak & Tadschikistan & Usbekistan & $400-2.500$ & $\begin{array}{l}99 \% \text { usbekisch, } \\
1 \% \text { kirgisisch }\end{array}$ \\
\hline Schachimardan & Usbekistan & Kirgistan & 5.000 & $\begin{array}{l}91 \% \text { usbekisch, } \\
9 \% \text { kirgisisch }\end{array}$ \\
\hline Soch & Usbekistan & Kirgistan & $25.000-70.000$ & $\begin{array}{c}99 \% \text { tadschikisch, } \\
1 \% \text { kirgisisch }\end{array}$ \\
\hline Woruch & Tadschikistan & Kirgistan & $10.000-30.000$ & $\begin{array}{l}95 \% \text { tadschikisch, } \\
5 \% \text { kirgisisch }\end{array}$ \\
\hline Kairagach & Tadschikistan & Kirgistan & 150 & $100 \%$ tadschikisch \\
\hline
\end{tabular}

Quelle: Taschtemchanowa, R. et al. (2015): 521; <http://krepublishers.com/02-Journals/T-Anth/Anth-22-0-000-15-Web/Anth-223-000-15-Abst-PDF/T-ANTH-SV-22-3-518-15-1538-Tashtemkhanova-R/T-ANTH-SV-22-3-518-15-1538-Tashtemkhanova-RTx[13].pdf> 


\section{Juni - 21. Juli 2017}

\section{Kasachstan}

\begin{tabular}{|c|c|}
\hline 24.6.2017 & $\begin{array}{l}\text { Der Vorsitzende der Stiftung "Schurnalisty w bede« (Journalisten in Not) und ehemalige Journalist, Ramasan } \\
\text { Jesergepow, schließt sich dem Hungerstreik des wegen seiner Rolle bei den Landprotesten im Frühjahr } 2016 \text { zu } \\
\text { fünf Jahren Haft verurteilten Bürgerrechtlers Maks Bokejew an. Bokejew protestiert bereits seit Anfang Juni } \\
\text { mit einer Verweigerung der Nahrungsaufnahme gegen seine Verlegung in ein anderes Gefängnis. }\end{array}$ \\
\hline 24.6 .2017 & $\begin{array}{l}\text { Die Enthüllung der kasachstanischen Flagge durch LGBT-Aktivisten bei der Gay Parade in New York sorgt für } \\
\text { heftige Debatten in den sozialen Netzwerken und Kritik führender kasachstanischer Politiker. }\end{array}$ \\
\hline 25.6 .2017 & $\begin{array}{l}\text { Fürst Albert II. von Monaco wird von Präsident Nursultan Nasarbajew zu bilateralen Gesprächen sowie einem } \\
\text { Rundgang über das EXPO-Gelände empfangen. }\end{array}$ \\
\hline 25.6 .2017 & $\begin{array}{l}\text { Die usbekischen Behörden lehnen die Auslieferung des wegen extremistischer Videos auf seinem Handy fest- } \\
\text { genommenen kasachstanischen Staatsbürgers Witalij Nentschenko an Kasachstan ab. }\end{array}$ \\
\hline 27.6.2017 & $\begin{array}{l}\text { Die NGO International Commission of Jurists ruft die kasachstanischen Behörden dazu auf, sich nicht in die } \\
\text { Arbeit unabhängiger Rechtsanwälte einzumischen. }\end{array}$ \\
\hline 28.6.2017 & $\begin{array}{l}\text { Nach Angaben des Analyseportals Ranking.kz hat die Regierung in den letzten fünf Jahren insgesamt } 565 \mathrm{Mrd} \text {. } \\
\text { Tenge (1,7 Mrd. US-Dollar) in die Organisation der EXPO-2017 investiert. }\end{array}$ \\
\hline 29.6 .2017 & $\begin{array}{l}\text { Die oppositionelle Website Kazakhstan } 2.0 \text { mit Sitz in London berichtet, dass am 28.6. die neue unabhängige } \\
\text { Journalistenvereinigung Media Union of Kazakhstan gegründet wurde. Es wird ein Zusammenhang mit der } \\
\text { befürchteten Auflösung des bestehenden Journalistenverbands durch die Behörden vermutet. }\end{array}$ \\
\hline 29.6 .2017 & $\begin{array}{l}\text { Auf Beschluss eines Gerichts in Almaty wird das Gebietsbüro der Zeugen Jehovas aus unbekannten Gründen } \\
\text { für drei Monate geschlossen und eine Strafe von } 680.000 \text { Tenge (ca. } 2.100 \text { US-Dollar) gegen die Religionsge- } \\
\text { meinschaft verhängt. }\end{array}$ \\
\hline 30.6 .2017 & $\begin{array}{l}\text { Der russische Präsident Wladimir Putin ratifiziert durch seine Unterschrift ein Protokoll, dass die Rückgabe } \\
\text { des bisher von der RF gepachteten } 300.000 \text { ha umfassenden Truppenübungsgeländes Emba (Gebiet Aktöbe) } \\
\text { von Russland an Kasachstan regelt. }\end{array}$ \\
\hline 2.7.2017 & $\begin{array}{l}\text { Präsident Nasarbajew unterschreibt ein Gesetz, mit dem Kasachstan Kirgistan eine Unterstützung in Höhe von } \\
100 \text { Mio. US-Dollar für die Integration in die Eurasische Wirtschaftsunion (EEU) gewährt. }\end{array}$ \\
\hline 5.7.2017 & $\begin{array}{l}\text { In einer Rede anlässlich des Tags der Hauptstadt erklärt Präsident Nasarbajew, dass Astana ein Zentrum der } \\
\text { Modernisierung Kasachstans wie der globalen Konfliktbewältigung sei. Zuvor hatte Nasarbajew das renovierte } \\
\text { Nationaldenkmal Baiterek wiedereröffnet. }\end{array}$ \\
\hline 5.7.2017 & $\begin{array}{l}\text { In Astana findet die fünfte Runde der Verhandlungen im Syrien-Konflikt unter Teilnahme von Vertretern der } \\
\text { Garantiemächte des Waffenstillstandsabkommens Iran, Türkei und Russland statt. Die Diplomaten können } \\
\text { sich nach harten Verhandlungen jedoch nicht auf die Einrichtung von Deeskalationszonen einigen. }\end{array}$ \\
\hline 5.7.2017 & $\begin{array}{l}\text { Die Untersuchungshaft des ehemaligen Ministers für die nationale Wirtschaft, Kuandyk Bischimbajew, wird } \\
\text { zum wiederholten Male verlängert, diesmal bis zum 10.8.2017. Bischimbajew ist seit Ende Dezember } 2016 \\
\text { wegen Korruptionsvorwürfen in Haft. }\end{array}$ \\
\hline 5.7.2017 & $\begin{array}{l}\text { Der Präsident der Internationalen Föderation der Gesellschaften des Roten Kreuzes/Roten Halbmonds (IFRC), } \\
\text { Tadateru Konoé, trifft zu einem Besuch in Astana ein, wo er u. a. von Außenminister Kairat Abdrachmanow } \\
\text { zu Gesprächen über die Zusammenarbeit empfangen wird. }\end{array}$ \\
\hline 5.7.2017 & $\begin{array}{l}\text { In Washington finden bilaterale Regierungsverhandlungen zur militärischen Zusammenarbeit zwischen } \\
\text { Kasachstan und USA in den Jahren 2018-22 statt. }\end{array}$ \\
\hline 6.7.2017 & $\begin{array}{l}\text { Aus dem Autonomen Gebiet Xinjiang der VR China stammende Kasachen rufen Präsident Nasarbajew in einer } \\
\text { Erklärung dazu auf, bei der chinesischen Führung gegen ihre Volksgruppe diskriminierende Maßnahmen zu } \\
\text { protestieren. Zuvor waren zahlreichen Kasachen mit chinesischer Staatsangehörigkeit beim Grenzübertritt nach } \\
\text { China ihre kasachstanischen Aufenthaltstitel entzogen worden. }\end{array}$ \\
\hline 6.7.2017 & $\begin{array}{l}\text { Energieminister Kanat Bosumbajew kündigt auf einer Energiemesse in Istanbul an, dass Kasachstan die im } \\
\text { Rahmen von OPEC-Verhandlungen vereinbarte Deckelung der Erdölfördermengen in den kommenden zwei } \\
\text { Monaten graduell aufgeben werde. }\end{array}$ \\
\hline 10.7.2017 & $\begin{array}{l}\text { Dem bekannten russischen Bürgerrechtler Witalij Ponomarjow wurde an der kasachstanisch-kirgisischen Grenze } \\
\text { die Einreise nach Kirgistan verweigert, meldet RFE/RL. }\end{array}$ \\
\hline
\end{tabular}




\begin{tabular}{|c|c|}
\hline 10.7.2017 & $\begin{array}{l}\text { Vier Mitarbeiter des Gesundheitsministeriums, darunter die Leiterin des pharmazeutischen Komitees, Larisa } \\
\text { Pak, werden wegen Korruptionsvorwürfen festgenommen. }\end{array}$ \\
\hline 10.7.2017 & $\begin{array}{l}\text { Die EBRD macht eine neue vierjährige Kasachstan-Strategie publik, die auf eine bessere Ausbalanzierung von } \\
\text { öffentlichem und privatem Sektor abzielt. }\end{array}$ \\
\hline 10.7.2017 & $\begin{array}{l}\text { Mit Unterschrift Präsident Nasarbajews treten Gesetzesänderungen in Kraft, die u. a. den Entzug der kasachstani- } \\
\text { schen Staatsbürgerschaft von im Ausland kämpfenden Terroristen ermöglichen und die Kandidatur von unab- } \\
\text { hängigen Präsidentschaftskandidaten einschränken auf Personen, die mindestens fünf Jahre im Staatsdienst } \\
\text { tätig waren. }\end{array}$ \\
\hline 11.7.2017 & $\begin{array}{l}\text { Bundespräsident Frank-Walter Steinmeier trifft zu einem Staatsbesuch in Astana ein, wo er u. a. von Präsi- } \\
\text { dent Nasarbajew zu Gesprächen über das bilaterale Verhältnis empfangen und am Nationentag Deutschland } \\
\text { im Rahmen der EXPO teilnehmen wird. }\end{array}$ \\
\hline 12.7.2017 & $\begin{array}{l}\text { Der kasachstanische Geschäftsmann Adil Tojganbajew, ehemaliger Schwiegersohn des kirgisischen Ex-Präsi- } \\
\text { denten Askar Akajew, wird von einem Gericht in Bischkek wegen Betrugs u. a. Wirtschaftsvergehen in absen- } \\
\text { tia zu } 20 \text { Jahren Haft und Konfiszierung des Eigentums verurteilt. }\end{array}$ \\
\hline 12.7.2017 & $\begin{array}{l}\text { Der wegen Umsturzplänen und mehrerer anderer Vergehen im November } 2016 \text { zu } 21 \text { Jahren Haft verurteilte } \\
\text { ehemalige Eigentümer der Brauerei von Schymkent (Gebiet Südkasachstan), Tochtar Toleschow, sieht sich vor } \\
\text { einem Gericht seiner Heimatstadt mit einem weiteren Prozess wegen Steuerhinterziehung und Schmiergeld- } \\
\text { zahlung konfrontiert. }\end{array}$ \\
\hline 13.7.2017 & $\begin{array}{l}\text { In Schymkent beginnt der Prozess gegen die ehemalige Vorsitzende der im letzten Winter aufgelösten Konfö- } \\
\text { deration unabhängiger Gewerkschaften, Larisa Charkowaja, wegen illegaler Aneignung und Verschwendung } \\
\text { von Haushaltsmitteln. }\end{array}$ \\
\hline 13.7.2017 & $\begin{array}{l}\text { In seiner Rede anlässlich des 25. Jahrestags der Gründung des Komitees für nationale Sicherheit (KNB) for- } \\
\text { dert Präsident Nasarbajew, sämtliche Bestrebungen zur Destabilisierung der Gesellschaft scharf zu verfolgen. }\end{array}$ \\
\hline 13.7.2017 & Die erste Elektro-Buslinie Kasachstans nimmt in Almaty im Testregime ihre Arbeit auf. \\
\hline 13.7.2017 & $\begin{array}{l}\text { Vier Senatoren, u. a. der ehemalige KNB-Chef Nurtaj Abykajew, werden auf Anweisung von Präsident Nasar- } \\
\text { bajew entlassen. }\end{array}$ \\
\hline 13.7.2017 & $\begin{array}{l}\text { Die größte kasachstanische Bank, Halyk Bank, deren Eigentümer der Schwiegersohn von Präsident Nasarba- } \\
\text { jew, Timur Kulibajew, ist, übernimmt für } 185 \text { Mrd. Tenge (ca. } 560 \text { Mio. US-Dollar) 96,8\% der Anteile der } \\
\text { Kazkommertsbank. }\end{array}$ \\
\hline 13.7.2017 & $\begin{array}{l}\text { Die Sprecherin des spanischen Parlaments, Ana Pastor, trifft in Astana mit Präsident Nasarbajew zu Gesprä- } \\
\text { chen über die bilaterale Zusammenarbeit zusammen und eröffnet den spanischen Pavillon auf der EXPO. }\end{array}$ \\
\hline 17.7.2017 & $\begin{array}{l}\text { Die türkischen Behörden setzen mehr als } 1.900 \text { Bürger Kasachstans wegen mutmaßlicher Kontakte zum IS auf } \\
\text { die Schwarze Liste derer, denen die Einreise untersagt wird. }\end{array}$ \\
\hline 17.7.2017 & $\begin{array}{l}\text { RIA Nowosti meldet, dass Interpol Ex-BTA Bank-Chef Muchtar Abljasow (der seit Jahren von Kasachstan und } \\
\text { Russland wegen des Verdachts der Unterschlagung von mehreren Mrd. US-Dollar gesucht, aber im Dezem- } \\
\text { ber } 2016 \text { in Frankreich auf freien Fuß gesetzt worden war) aus der Datenbank der international gesuchten Per- } \\
\text { sonen gelöscht hat. }\end{array}$ \\
\hline 18.7.2017 & $\begin{array}{l}\text { In Astana beginnt eine zweitägige Konferenz der CAREC-Staaten zur Förderung von neuen Technologien im } \\
\text { Bereich regenerativer Energiequellen. }\end{array}$ \\
\hline 18.7.2017 & $\begin{array}{l}\text { Verteidigungsminister Saken Schanusakow empfängt in Astana den Chef des Nationalen Sicherheitsrats Afgha- } \\
\text { nistans, Hanif Atmar, zu Gesprächen über die bilaterale Zusammenarbeit im Bereich der regionalen Sicherheit. }\end{array}$ \\
\hline 18.7.2017 & $\begin{array}{l}\text { Kulturminister Arystanbek Muchamediuly gibt die Gründung des neuen Staatsunternehmens KazakhTourism } \\
\text { bekannt, das sich um die Förderung des touristischen Potentials und die Akquise von Investitionen kümmern soll. }\end{array}$ \\
\hline 19.7.2017 & $\begin{array}{l}\text { Ein Militärgericht in Astana verurteilt fünf ehemalige Mitarbeiter des Verteidigungs- und Finanzministeriums } \\
\text { sowie sechs Geschäftsleute wegen Korruption zu Gefängnisstrafen zwischen drei und zwölf Jahren. }\end{array}$ \\
\hline 19.7.2017 & $\begin{array}{l}\text { Die Verwaltung der grenzüberschreitenden Sonderwirtschaftszone Chorgos meldet, dass dort in der ersten Jah- } \\
\text { reshälfte } 2017 \text { ca. 1,6 Mio. Besucher gezählt wurden. }\end{array}$ \\
\hline 19.7.2017 & $\begin{array}{l}\text { Der Chef der Präsidentenadministration, Adilbek Schaksybekow, wird für fünf weitere Jahre in seinem Amt } \\
\text { bestätigt. }\end{array}$ \\
\hline
\end{tabular}




\begin{tabular}{|c|c|}
\hline 24.6.2017 & $\begin{array}{l}\text { Das Gebietsgericht von Osch verurteilt einen Bewohner des Bezirks Arawan zu einer achtjährigen Freiheits- } \\
\text { strafe wegen Mitgliedschaft im IS. Der Mann soll } 2013 \text { seine Landsleute zum Dschihad und einem Umsturz } \\
\text { in Kirgistan aufgerufen haben. }\end{array}$ \\
\hline 25.6 .2017 & $\begin{array}{l}\text { In Kotschkor (Gebiet Naryn) kommt eine deutsche Touristin durch einen Verkehrsunfall ums Leben, zwei } \\
\text { weitere werden schwer verletzt. }\end{array}$ \\
\hline 25.6.2017 & $\begin{array}{l}\text { Im usbekischen Andischan endet eine Sitzung der kirgischen und usbekischen Regierungsarbeitsgruppen zur } \\
\text { Delimitierung und Demarkation der gemeinsamen Staatsgrenze. }\end{array}$ \\
\hline 26.6.2017 & $\begin{array}{l}\text { Der kirgisische Dienst von RFE/RL meldet, dass zwei kirgisische Internetnutzer wegen Anheizens nationalen } \\
\text { Hasses verhaftet wurden, die sich in den sozialen Netzwerken abfällig über die Flagge Kirgistans geäußert hatten. }\end{array}$ \\
\hline 27.6.2017 & $\begin{array}{l}\text { Angehörige der Grenztruppen Chinas und Kirgistans führen im Autonomen Kirgisischen Bezirk Kysylsu im } \\
\text { Autonomen Gebiet Xinjiang (China) die gemeinsame Anti-Terror-Übung Tian-Schan 3-2017 durch. }\end{array}$ \\
\hline 27.6.2017 & $\begin{array}{l}\text { Ein Gericht in Bischkek verurteilt zwei Männer und eine Frau wegen Mittäterschaft an einem Attentat auf die } \\
\text { chinesische Botschaft zu Freiheitsstrafen von zehn bis } 18 \text { Jahren. Am 30.8.2017 war ein Selbstmordattentäter } \\
\text { mit einem Auto in das Tor der Botschaft gerast und hatte eine Bombe gezündet, drei Personen wurden verletzt, } \\
\text { es entstand großer Sachschaden. Als Auftraggeber wird eine in Syrien kämpfende Uighurengruppe vermutet. }\end{array}$ \\
\hline 28.6.2017 & $\begin{array}{l}\text { Präsident Almasbek Atambajew empfängt den Vorsitzenden der russischen Duma, Wjatscheslaw Wolodin, zu } \\
\text { Gesprächen über das bilaterale Verhältnis. Wolodin trifft auch mit dem Sprecher des kirgisischen Parlamentes, } \\
\text { Tschynybaj Tursunbekow, und Abgeordneten zusammen. }\end{array}$ \\
\hline 28.6.2017 & $\begin{array}{l}\text { Das Staatskomitee für nationale Sicherheit teilt mit, dass im Rahmen von Anti-Terror-Maßnahmen vier kir- } \\
\text { gisische Staatsbürger, die einen Anschlag vorbereitet haben sollen, wegen des Verdachts der Zugehörigkeit zu } \\
\text { einer internationalen Terrororganisation verhaftet wurden. Genauere Angaben über Zeitpunkt und Ort der } \\
\text { Verhaftung wurden nicht gemacht. }\end{array}$ \\
\hline 28.6.2017 & $\begin{array}{l}\text { Nach Angaben aus dem Ministerium für Katastrophenschutz hat die EBRD } 16 \text { Mio. US-Dollar für die Reha- } \\
\text { bilitierung der Uranminen auf kirgisischem Territorium bewilligt. }\end{array}$ \\
\hline 28.6.2017 & $\begin{array}{l}\text { Seit } 2012 \text { hat der Bergbausektor 6,5 Mrd. Som (94,4 Mio. US-Dollar) zum Staatsbudget beigetragen, allein } \\
2016 \text { 1,2 Mrd. Som (17,4 Mio. US-Dollar), teilt der Vorsitzende des Staatskomitees für Industrie, Energie und } \\
\text { Bergbau, Dujschenbek Silalijew, vor dem Parlament mit. }\end{array}$ \\
\hline 29.6 .2017 & $\begin{array}{l}\text { Im Prozess gegen den Parteichef von Ata-Meken, Omurbek Tekebajew, wird Leonid Majewskij, Eigentümer } \\
\text { mehrerer russischer Telekommunikationsfirmen, als Zeuge der Anklage befragt. Das Verfahren beruht auf } \\
\text { Majewskijs Aussage, dass er Tekebajew } 2010 \text { in seiner Eigenschaft als stellvertretender Regierungschef der } \\
\text { Übergangsregierung } 1 \text { Mio. US-Dollar für den Zugang zum kirgisischen Telekommunikationsmarkt gezahlt } \\
\text { habe (, ohne dass Tekebajew eine Gegenleistung erbrachte). }\end{array}$ \\
\hline 30.6 .2017 & $\begin{array}{l}\text { Ein Gericht in Bischkek entspricht dem Antrag der Generalstaatsanwaltschaft bezüglich der Verteidigung der } \\
\text { Ehre und Würde Präsident Atambajews gegen die Direktorin der Rechtsklinik Adilet, Tscholpon Dschaku- } \\
\text { powa, den Journalisten Naryn Ajyp, die Chefredakteurin von Zanoza.kg, Dina Maslowa und den Fonds Pro- } \\
\text { Media. Ein inkriminierter Artikel muss von der Website entfernt werden und die vier Genannten müssen für } \\
\text { den entstandenen moralischen Schaden eine Kompensation von je } 3 \text { Mio. Som (43.600 US-Dollar) zahlen. }\end{array}$ \\
\hline 3.7.2017 & $\begin{array}{l}\text { Ca. } 30 \text { Bewohner des Dorfes Tschandalasch, Bezirk Tschatkal (Gebiet Dschalalabad), blockieren aus Protest } \\
\text { gegen Umweltbelastungen durch den Goldabbau die Maschinen der Firma Konsalidit Awrum. Die Firma hatte } \\
\text { im Frühjahr } 2017 \text { mit der Förderung im Bezirk Tschatkal begonnen. }\end{array}$ \\
\hline 4.7.2017 & $\begin{array}{l}\text { Parlamentssprecher Tursunbekow erörtert in Bischkek mit der Vorsitzenden des Föderationsrates des russischen } \\
\text { Parlamentes, Walentina Matwijenko, diverse Aspekte der Zusammenarbeit beider Volksvertretungen. Am Vor- } \\
\text { tag hatte Präsident Atambajew Matwijenko zu Gesprächen empfangen. }\end{array}$ \\
\hline 6.7.2017 & $\begin{array}{l}\text { Premier Sooronbaj Dscheenbekow nimmt am CASA-1000-Gipfel in Duschanbe teil. Am Rande der Veranstal- } \\
\text { tung trifft er mit dem tadschikischen Präsidenten Emomali Rachmon zu Gesprächen zusammen und erörtert } \\
\text { mit dem afghanischen Präsidenten Ashraf Ghani u. a. die Situation der Kirgisen in Afghanistan. }\end{array}$ \\
\hline 6.7.2017 & $\begin{array}{l}\text { Das Innenministerium meldet die Verhaftung von mehreren der Mitgliedschaft in der extremistischen Hizb } \\
\text { ut-Tahrir verdächtigen Personen im Gebiet Dschalalabad. }\end{array}$ \\
\hline 6.7.2017 & $\begin{array}{l}\text { RFE/RL meldet die Verhaftung von zwei kirgisischen Staatsbürgern unter dem Verdacht der Anwerbung für } \\
\text { terroristische Organisationen durch das Internet. Sie sollen zwei Männer nach Syrien vermittelt haben, wo } \\
\text { einer von ihnen zu Tode kam. }\end{array}$ \\
\hline
\end{tabular}




\begin{tabular}{|c|c|}
\hline 6.7.2017 & $\begin{array}{l}\text { Nach Angaben der nationalen Energiegesellschaft hat Kirgistan am 16.6. mit Stromlieferungen an Usbekistan } \\
\text { begonnen. Bis zum 4.7. wurden bereits 192,5 Mio. kWh geliefert. }\end{array}$ \\
\hline 7.7.2017 & $\begin{array}{l}\text { Mehrere Feministinnen versammeln sich leicht bekleidet in der Nähe der Hauptmoschee von Bischkek zu einer } \\
\text { friedlichen Protestaktion unter dem Motto "Schreibt uns nicht vor, wie wir uns anzuziehen haben". }\end{array}$ \\
\hline 9.7.2017 & $\begin{array}{l}\text { Dem bekannten russischen Menschenrechtler Witalij Ponomarjow von Memorial wird die Einreise nach Kir- } \\
\text { gistan verweigert. Ponomarjow hatte vom 30.6. bis 3.7. an einer Menschenrechtsveranstaltung in Bischkek } \\
\text { teilgenommen und war im Anschluss daran nach Astana geflogen. Die Wiedereinreise nach Kirgistan soll ihm } \\
\text { nach Angaben mehrerer Medien aufgrund eines geheimen Briefes des Staatskomitees für nationale Sicherheit } \\
\text { verwehrt worden sein. }\end{array}$ \\
\hline 10.7.2017 & $\begin{array}{l}\text { Die Kumtor Gold Company teilt mit, dass bei einem Unfall am Vortag, bei dem ein Tankwagen auf dem Weg } \\
\text { zur Goldmine umgestürzt war, ca. } 7 \text { t Dieseltreibstoff ausgelaufen sind. Das Gelände sei am gleichen Tag gerei- } \\
\text { nigt worden, Wasserverschmutzungen nicht zu erwarten. }\end{array}$ \\
\hline 10.7.2017 & $\begin{array}{l}\text { In Gegenwart von Präsident Atambajew wird der Vertrag über den Bau der Staustufe für das Wasserkraftwerk } \\
\text { am oberen Naryn zwischen der kirgisischen Regierung und der weithin unbekannten tschechischen Liglass } \\
\text { TradingCZ, SRO unterzeichnet. }\end{array}$ \\
\hline 10.7.2017 & $\begin{array}{l}\text { Der bisherige stellvertretende Vorsitzende des staatlichen Grenzdienstes, Ularbek Scharschijew, wird dessen } \\
\text { neuer Vorsitzender. }\end{array}$ \\
\hline 10.7.2017 & $\begin{array}{l}\text { Der Schweizer Finanzminister Ueli Maurer trifft während seines Bischkekbesuches mit seinem Amtskollegen } \\
\text { Adylbek Kasymalijew sowie Nationalbankchef Tolkunbek Abdygulow zusammen und wird auch von Präsi- } \\
\text { dent Atambajew empfangen. }\end{array}$ \\
\hline 11.7.2017 & $\begin{array}{l}\text { Nach Angaben aus der Verwaltung für Demographie und Statistik hatte Kirgistan Anfang } 2017 \text { 6,14 Mio. } \\
\text { Einwohner. }\end{array}$ \\
\hline 11.7.2017 & $\begin{array}{l}\text { Im ersten Halbjahr } 2017 \text { betrug das BIP Kirgistans nach Angaben des Nationalen statistischen Komitees } 194 \\
\text { Mrd. Som (2,8 Mrd. US-Dollar), d. i. ein Wachstum von 6,4\%. Der Goldexport stieg im gleichen Zeitraum } \\
\text { um } 41 \% \text {. }\end{array}$ \\
\hline 11.7.2017 & $\begin{array}{l}\text { In Bischkek findet eine gemeinsame Sitzung der Arabischen Koordinationsgruppe und der kirgisischen Regie- } \\
\text { rung statt, bei der die Förderung von zehn Projekten im Transport-, Gesundheits- und Landwirtschaftssektor } \\
\text { mit einem Gesamtvolumen von } 357 \text { Mio. US-Dollar beschlossen wird. }\end{array}$ \\
\hline 12.7 .2017 & $\begin{array}{l}\text { Präsident Atambajew empfängt eine US-Wirtschaftsdelegation, der Vertreter von Baker \& McKenzie LLP, } \\
\text { General Electric, AGCO Corporation, Coca-Cola, John Deere u. a. angehören. }\end{array}$ \\
\hline 12.7 .2017 & $\begin{array}{l}\text { Der kasachstanische Geschäftsmann Adil Tojganbajew, ehemaliger Schwiegersohn von Ex-Präsident Askar } \\
\text { Akajew, wird von einem Gericht in Bischkek wegen Betrugs u. a. Wirtschaftsvergehen in absentia zu } 20 \text { Jah- } \\
\text { ren Haft und Konfiszierung des Eigentums verurteilt. }\end{array}$ \\
\hline 13.7.2017 & $\begin{array}{l}\text { Interfax meldet, dass die Polizei im Gebiet Naryn den Versuch von ca. } 40 \text { Männern vereitelt hat, Golderz aus } \\
\text { einem Lager von Kyrgyzaltyn an der Goldmine Solton-Sary zu rauben und } 23 \text { Personen festgenommen wurden. }\end{array}$ \\
\hline 13.7.2017 & $\begin{array}{l}\text { Mitarbeiter des Staatskomitees für nationale Sicherheit haben im Rahmen von Anti-Terror-Maßnahmen einen } \\
\text { der Mitgliedschaft im IS Verdächtigen festgenommen. Er soll in Syrien und Irak gekämpft haben und mit dem } \\
\text { Ziel der Bildung einer Untergrundzelle nach Kirgistan zurückgekehrt sein. }\end{array}$ \\
\hline 13.7.2017 & $\begin{array}{l}\text { Im Prozess gegen den Ata-Meken Führer Tekebajew treten Ex-Präsidentin Rosa Otunbajewa, das ehemalige } \\
\text { Mitglied der Übergangsregierung Emilbek Kaptagajew und weitere prominente Politiker als Zeugen der Ver- } \\
\text { teidigung auf. }\end{array}$ \\
\hline 14.7 .2017 & $\begin{array}{l}\text { RFE/RL berichtet, dass die Islamische Universität in Bischkek vom Ministerium für Bildung und Wissenschaft } \\
\text { eine Lizenz für Lehrtätigkeit im Bereich Theologie erhalten hat. Die bereits } 1990 \text { gegründete Hochschule hatte } \\
\text { bislang ohne offizielle Registrierung durch das Ministerium gearbeitet. }\end{array}$ \\
\hline 17.7.2017 & $\begin{array}{l}\text { Nach Angaben der Nachrichtenagentur UzTAG kamen in Taschkent erstmals seit neun Jahren Vertreter der } \\
\text { Luftfahrtbehörden Kirgistans und Usbekistans zu Gesprächen zusammen. }\end{array}$ \\
\hline 18.7.2017 & $\begin{array}{l}\text { Während eines Treffens Gesundheitsministers Talantbek Batyralijews mit seinem iranischen Amtskollegen } \\
\text { Seyyed Hassan Ghazizadeh Hashemi in Bischkek werden eine Reihe von Dokumenten über eine bilaterale } \\
\text { Zusammenarbeit unterzeichnet. }\end{array}$ \\
\hline 19.7.2017 & $\begin{array}{l}\text { Nach Angaben aus dem Gesundheitsministerium steigt die Zahl der HIV-Infektionen nach wie vor jährlich } \\
\text { um ca. } 10 \% \text {. Am } 1.6 .2017 \text { waren knapp } 7.500 \text { HIV-positv Getestete registriert, davon } 595 \text { Kinder. An AIDS } \\
\text { erkrankt waren } 902 \text { Personen. }\end{array}$ \\
\hline
\end{tabular}




\begin{tabular}{|l|l|}
\hline 20.7.2017 & $\begin{array}{l}\text { In seiner Rede auf dem Internationalen Forum »Altai-Zivilisation und der Völker mit altaiischen Sprachen« } \\
\text { spricht sich Präsident Atambajew zum wiederholten Male gegen einen Übergang der Schreibweise des Kirgisi- } \\
\text { schen in lateinische Schrift aus. Am Rande der Veranstaltung empfängt Atambajew die Präsidenten der Auto- } \\
\text { nomen Republiken der RF Tatarstan und Baschkortostan, Rustam Minnichanow und Rustem Chamitow, zu } \\
\text { Gesprächen über das bilaterale Verhältnis. }\end{array}$ \\
\hline 20.7.2017 & $\begin{array}{l}\text { Nach Regierungsangaben hatte der Tourismus 2016 einen Anteil am BIP von 4,7\% (21,4 Mrd. Som = 310,8 Mio. } \\
\text { US-Dollar). }\end{array}$ \\
\hline 21.7.2017 & $\begin{array}{l}\text { Bei ihrer zweiten gemeinsamen Konferenz in Tscholpon-Ata (Gebiet Issyk-Kul) vereinbaren die Innenminis- } \\
\text { ter Kirgistans und Kasachstans, Ulan Israilow und Kalmuchanbet Kasymow, eine intensivere Zusammenar- } \\
\text { beit im Kampf gegen den Extremismus. }\end{array}$ \\
& $\begin{array}{l}\text { Das Innenministerium gibt die Verhaftung eines extremistischer Tätigkeit Verdächtigen in Osch bekannt. Der } \\
\text { Mann soll IS-Mitglied sein und in Syrien gekämpft haben, bei einer Haussuchung wurden Sprengstoff und } \\
\text { islamistische Literatur gefunden. }\end{array}$ \\
\hline 21.7.2017 & $\begin{array}{l}\text { Nach Angaben der Zentralen Wahlkommission haben sich bislang 35 Personen um die Nominierung als Kan- } \\
\text { didat für die Präsidentschaftswahlen am 15.10.2017 beworben, darunter Premier Dscheenbekow (SDPK), } \\
\text { der in Untersuchungshaft sitzende Ata-Meken Führer Tekebajew (als unabhängiger Kandidat), die ehemali- } \\
\text { gen Premiers Temir Sarijew und Omurbek Babanow, aber auch Rentner und Arbeitslose. Die Bewerbungs- } \\
\text { frist endet am 1.8.2017. }\end{array}$ \\
\hline
\end{tabular}

\section{Tadschikistan}

\begin{tabular}{|c|c|}
\hline 26.6.2017 & $\begin{array}{l}\text { Erst jetzt wird bekannt, dass die Regierung vor einigen Wochen die Bildung einer Staatsagentur für Ernäh- } \\
\text { rungssicherheit beschlossen hat. }\end{array}$ \\
\hline 27.6 .2017 & $\begin{array}{l}\text { Am heutigen Tag der nationalen Einheit wird des 20. Jahrestages der Unterzeichnung des Friedensvertrages } \\
\text { zwischen Präsident Emomali Rachmon und dem Führer der Vereinigten Tadschikischen Opposition, Said } \\
\text { Abdullo Nuri, in Moskau gedacht. Die zentrale Feier findet in der Nähe von Duschanbe in Wachdat (Gebiete } \\
\text { unter republikanischer Verwaltung) statt. Vertreter der damaligen Opposition sind nicht eingeladen. }\end{array}$ \\
\hline 27.6.2017 & $\begin{array}{l}\text { Der tadschikische Dienst von RFE/RL meldet, dass ein 26jähriger tadschikischer Staatsbürger, der nach dem } \\
\text { Putschversuch in der Türkei als Anhänger der Gülen-Bewegung verhaftet worden war, freigelassen wurde. }\end{array}$ \\
\hline 28.6.2017 & $\begin{array}{l}\text { Am Rande einer regulären Sitzung der Innenminister der GUS-Staaten in Duschanbe findet mit einem Gespräch } \\
\text { zwischen Ramason Rahimsoda und Abdusalom Asisow das erste bilaterale Treffen zwischen den Innenminis- } \\
\text { tern Tadschikistans und Usbekistans seit } 19 \text { Jahren statt. Gesprächsgegenstand sind transnationale organisierte } \\
\text { Kriminaltität, Terrorismus und Drogenhandel. }\end{array}$ \\
\hline 29.6 .2017 & $\begin{array}{l}\text { Asia-Plus meldet erst jetzt, dass Präsident Rachmon bereits am 7.6. ein Dekret über die Bildung einer Begna- } \\
\text { digungskommission unterzeichnet hat. Die neue Institution, die am 1.9.2017 die Arbeit aufnehmen wird, soll } \\
\text { den Präsidenten mit Vorschlägen für Amnestien beratend unterstützen. }\end{array}$ \\
\hline 29.6 .2017 & $\begin{array}{l}\text { Der ungarische Außenminister Péter Szijjártó wird in Duschanbe von Präsident Rachmon und seinem Amts- } \\
\text { kollegen Sirodschiddin Aslow sowie Parlamentssprecher Schukurdschon Suchurow zu Gesprächen über eine } \\
\text { Intensivierung der bilateralen Zusammenarbeit und internationale Fragen empfangen. }\end{array}$ \\
\hline 29.6 .2017 & $\begin{array}{l}\text { Die Botschaft Irans schließt auf Forderung der tadschikischen Behörden ihre Handels- und Kulturvertretung } \\
\text { in der Hauptstadt des Gebiets Sogd, Chudschand. }\end{array}$ \\
\hline 30.6 .2017 & $\begin{array}{l}\text { Das Unterhaus des Parlamentes schafft einstimmig eine im Oktober } 2016 \text { eingeführte Gesetzesänderung ab, } \\
\text { die es den Organen der Staatssicherheit erlaubt hatte, im Fall eines Terrorverdachtes auch ohne richterlichen } \\
\text { Beschluss Haussuchungen vorzunehmen. }\end{array}$ \\
\hline 2.7.2017 & $\begin{array}{l}\text { Bei einem Feuer auf dem größten Basar Tadschikistans, Korwon, werden } 25 \text { Läden und } 160 \text { Verkaufsstände } \\
\text { zerstört. }\end{array}$ \\
\hline 4.7.2017 & $\begin{array}{l}\text { Ein im Mai in der Autonomen Republik Adygej der RF verhafteter Neffe des zum IS übergelaufenen ehemali- } \\
\text { gen OMON-Chefs Gulmurod Chalimow wurde auf tadschikisches Ersuchen in der Vorwoche nach Tadschi- } \\
\text { kistan ausgeliefert. Ihm wird Anwerbung für eine terroristische Organisation vorgeworfen. Zwei Brüder und } \\
\text { zwei weitere Verwandte von Chalimow werden in der Nacht in der Nähe des Dorfes Ibrati, Bezirk Wose (Gebiet } \\
\text { Chatlon), während einer Schießerei mit Polizisten erschossen, drei weitere Verwandte festgenommen. Sie sol- } \\
\text { len versucht haben, illegal die Grenze nach Afghanistan zu überqueren. }\end{array}$ \\
\hline
\end{tabular}




\begin{tabular}{|c|c|}
\hline 5.7.2017 & $\begin{array}{l}\text { Während eines bilateralen Treffens Präsident Rachmons mit dem pakistanischen Premierminister Mohammed } \\
\text { Nawaz Sharif in Duschanbe werden mehrere Dokumente über die weitere Zusammenarbeit zwischen beiden } \\
\text { Staaten unterzeichnet. }\end{array}$ \\
\hline 6.7.2017 & $\begin{array}{l}\text { In der Regierungsresidenz Pugus nahe Duschanbe empfängt Präsident Rachmon seinen afghanischen Amtskol- } \\
\text { legen Ashraf Ghani und den pakistanischen Premier Sharif sowie den kirgisischen Premier Sooronbaj Dscheen- } \\
\text { bekow zu Gesprächen über Möglichkeiten der Beschleunigung des CASA-1000 Projektes. Am Rande der Ver- } \\
\text { anstaltung empfängt Rachmon den kirgisischen Premier und den afghanischen Präsidenten auch zu bilatera- } \\
\text { len Gesprächen. }\end{array}$ \\
\hline 6.7.2017 & $\begin{array}{l}\text { Asia-Plus meldet, dass ein Gericht in Duschanbe Bechrus Chalimow, den 18jährigen Sohn des zum IS über- } \\
\text { gelaufenen Ex-Omon-Chefs bereits vor ca. zwei Wochen zu einer zehnjährigen Freiheitsstrafe wegen Vorberei- } \\
\text { tung einer Straftat und Anwerbung von Kämpfern für den IS verurteilt hat. }\end{array}$ \\
\hline 9.7.2017 & $\begin{array}{l}\text { Vertreter der seit Herbst } 2015 \text { in Tadschikistan verbotenen Partei der Islamischen Wiedergeburt (darunter auch } \\
\text { ihr Führer Muchiddin Kabiri) und der Gruppe } 24 \text { gedenken mit einer Veranstaltung in Dortmund des 20. Jah- } \\
\text { restages der Unterzeichnung des tadschikischen Friedensabkommens. }\end{array}$ \\
\hline 10.7.2017 & $\begin{array}{l}\text { Das Ministerium für Bildung und Wissenschaft dementiert Berichte, dass es im Iran studierenden tadschiki- } \\
\text { schen Studenten die Ausreise zur Fortsetzung ihres Studiums verbiete. Zuvor hatten mehrere Eltern Medien- } \\
\text { vertretern mitgeteilt, dass ihre Kinder nach den Semesterferien an der Rückkehr an ihren iranischen Studien- } \\
\text { ort gehindert worden seien. }\end{array}$ \\
\hline 11.7.2017 & $\begin{array}{l}\text { In den Buchläden von Duschanbe taucht ein Buch Präsident Rachmons mit dem Titel »Weise Gedanken und } \\
\text { Aphorismen des Präsidenten Tadschikistans, Stifter des Friedens und der nationalen Einheit, Führers der Nation, } \\
\text { Emomali Rachmon« auf, das Äußerungen des Präsidenten zu Unabhängigkeit und Verfassung des Landes, } \\
\text { nationaler Identität, Geschichte, Sprache usw. enthält. }\end{array}$ \\
\hline 11.7.2017 & $\begin{array}{l}\text { In vielen Orten im Autonomen Gebiet Berg-Badachschan (GBAO) wie auf der ganzen Welt wird das diaman- } \\
\text { tene Jubiläum, d. h. der 60. Jahrestag der Amtsübernahme, des Aga-Khan, des geistlichen Führers der Ismai- } \\
\text { liten, feierlich begangen. }\end{array}$ \\
\hline 11.7.2017 & $\begin{array}{l}\text { Bildungsminister Nuriddin Said kündigt vor Journalisten die schrittweise Einführung der zwölfjährigen allge- } \\
\text { meinen Schulbildung ab } 2020 \text { an. Die Verlängerung der Schulzeit war schon für } 2016 \text { geplant gewesen, musste } \\
\text { aber aus wirtschaftlichen Gründen verschoben werden. }\end{array}$ \\
\hline 12.7.2017 & $\begin{array}{l}\text { Präsident Rachmon empfängt den Chef des Generaldirektorats für internationale Zusammenarbeit und Ent- } \\
\text { wicklung der Europäischen Kommission, Stefano Manservisi, zu Gesprächen über Stand und Perspektiven der } \\
\text { Zusammenarbeit. Manservisi besucht auch gemeinsam mit dem Sonderbeauftragten der EU für Zentralasien, } \\
\text { Peter Burian, und Vertretern der KfW und des Aga-Khan-Fonds den Bezirk Schamsiddin Schochin (Gebiet } \\
\text { Chatlon) an der Grenze zu Afghanistan. }\end{array}$ \\
\hline 13.7.2017 & Die russische Luftwaffe verlegt zwei Einheiten für gemeinsame Übungen nach Tadschikistan. \\
\hline 14.7 .2017 & $\begin{array}{l}\text { Nach Angaben des staatlichen Dienstes für Arbeitssicherheit kam in den ersten sechs Monaten } 2017 \text { bei den } \\
\text { Bauarbeiten für das Wasserkraftwerk Rogun ein Arbeiter ums Leben, sechs wurden verletzt. }\end{array}$ \\
\hline 14.7.2017 & $\begin{array}{l}\text { Präsident Rachmon empfängt den turkmenischen Außenminister Raschid Meredow zu Gesprächen über Stand } \\
\text { und Perspektiven des bilateralen Verhältnisses. }\end{array}$ \\
\hline 14.7.2017 & $\begin{array}{l}\text { Präsident Rachmon empfängt den Vorsitzenden des Rates der Unternehmer USA-Tadschikistan, Philip de Leon, } \\
\text { der mit Vertretern großer amerikanischer Konzerne wie General Electric, Coca-Cola, Visa, APR Energy Tad- } \\
\text { schikistan besucht. }\end{array}$ \\
\hline 18.7.2017 & $\begin{array}{l}\text { Im Gebiet Sogd wird ein 28jähriger Mann wegen Werbung für den IS zu einer achtjährigen Freiheitsstrafe } \\
\text { verurteilt. }\end{array}$ \\
\hline 18.7.2017 & $\begin{array}{l}\text { HRW und das Norwegische Helsinki-Komitee beklagen in einer Erklärung die Verfolgung von Familienan- } \\
\text { gehörigen oppositioneller Politiker in Tadschikistan. Anlass ist die Verfolgung von zehn Verwandten tadschi- } \\
\text { kischer Teilnehmer der oppositionellen Veranstaltung zum 20. Jahrestag der Unterzeichnung des Friedensab- } \\
\text { kommens in Dortmund am 9.7.2017. Das Innenministerium erklärt, dass es sich um Vorladungen, nicht Ver- } \\
\text { haftungen gehandelt habe. }\end{array}$ \\
\hline 18.7.2017 & $\begin{array}{l}\text { Eine der Töchter Präsident Rachmons, Zarina (23), wurde zur stellvertretenden Direktorin der größten Geschäfts- } \\
\text { bank Tadschikistans, der Orienbank, ernannt, melden mehrere Medien. Chef des Unternehmens ist Rachmons } \\
\text { Schwager Hasan Asadullosoda. }\end{array}$ \\
\hline
\end{tabular}




\begin{tabular}{|l|l|}
\hline 19.7.2017 & $\begin{array}{l}\text { Der Chef der Agentur für Drogenkontrolle, Scherchon Salimsoda, erörtert bei einem Treffen in Duschanbe } \\
\text { mit dem Generalstaatsanwalt von Belarus, Aleksandr Konjuk, die Situation des illegalen Drogenhandels aus } \\
\text { Afghanistan und Maßnahmen seiner Bekämpfung. }\end{array}$ \\
\hline 20.7.2017 & $\begin{array}{l}\text { Bei einem in der ersten Jahreshälfte 2017 insgesamt gesunkenen Handelsvolumen hat sich der Warenaustausch } \\
\text { mit Usbekistan im gleichen Zeitraum im Vergleich zum Vorjahr mit knapp 60 Mio. US-Dollar verdoppelt. }\end{array}$ \\
\hline 21.7.2017 & $\begin{array}{l}\text { Nach Angaben von Innenminister Rachimsoda konnten im ersten Halbjahr 2017 in Tadschikistan 12 Terror- } \\
\text { akte vereitelt werden. 228 Personen wurden unter dem Verdacht der Mitgliedschaft in einer terroristischen Ver- } \\
\text { einigung verhaftet, 13 tadschikische Staatsbürger kehrten freiwillig vom IS zurück. }\end{array}$ \\
\hline 21.7.2017 & $\begin{array}{l}\text { RFE/RL meldet, dass im Gebiet Chatlon ein Mensch an Gelbfieber gestorben ist, bei zwei weiteren besteht } \\
\text { Gelbfieberverdacht. }\end{array}$ \\
\hline 21.7.2017 & $\begin{array}{l}\text { Nach Angaben von Kulturminister Schamsuddin Omurbeksoda gegenüber Journalisten wurde von der Regie- } \\
\text { rung eine Kommission gebildet, die unter Berücksichtigung von Traditionen und modernen Erfordernissen } \\
\text { Kleidungsvorschläge für die Bürger des Landes erarbeiten soll. }\end{array}$ \\
\hline
\end{tabular}

\section{Turkmenistan}

\begin{tabular}{|c|c|}
\hline 26.6.2017 & $\begin{array}{l}\text { Der neue turkmenische Mobilfunkanbieter Ai Nasar übernimmt ab September } 2017 \text { das bisherige russische } \\
\text { Unternehmen MTC-Turkmenistan, dessen Lizenz auszulaufen droht. }\end{array}$ \\
\hline 28.6.2017 & In Tokio finden japanisch-turkmenische Regierungskonsultationen für Handels- und Wirtschaftsfragen statt. \\
\hline 28.6 .2017 & $\begin{array}{l}\text { Die staatlichen Fluglinien Turkmenchovajollary und Uzbekiston Havo Yullari unterzeichnen einen Vertrag } \\
\text { über die Wiederaufnahme von Direktflügen zwischen Taschkent und Aschchabad bis zum Ende des Jahres. }\end{array}$ \\
\hline 29.6 .2017 & $\begin{array}{l}\text { Anlässlich des 60. Geburtstags von Präsident Gurbanguly Berdymuchammedow finden im ganzen Land Feiern } \\
\text { statt. In der Machtumkuli-Universität Aschchabad wird das Buch »Die glückliche Epoche des Führers vorgestellt. }\end{array}$ \\
\hline 3.7 .2017 & $\begin{array}{l}\text { Der afghanische Präsident Ashraf Ghani wird in Aschchabad von seinem turkmenischen Amtskollegen Ber- } \\
\text { dymuchammedow zu Gesprächen über das bilaterale Verhältnis empfangen. Ghani und Berdymuchamme- } \\
\text { dow unterzeichnen mehrere Verträge über strategische Partnerschaften in den Bereichen Energie, Transport } \\
\text { und Wissenschaft. }\end{array}$ \\
\hline 6.7.2017 & $\begin{array}{l}\text { Präsident Berdymuchammedow weist im Rahmen eines Treffens des Nationalen Sicherheitsrates den Chef des } \\
\text { Migrationsdienstes, Meilis Nobatow, an, während der Asien-Spiele im September die Ein- und Ausreise von } \\
\text { Ausländern streng zu überwachen. }\end{array}$ \\
\hline 7.7.2017 & $\begin{array}{l}\text { Während der letzten Kabinettssitzung vor der Sommerpause entlässt Präsident Berdymuchammedow Finanz- } \\
\text { minister Muchammetguly Muchammedow und Bau- und Architekturminister Tschary Atajew wegen schwerer } \\
\text { Nachlässigkeiten in der Amtsführung. Nachfolger werden Gotschmyrad Muradow und Tscharyjar Achmedow. }\end{array}$ \\
\hline 9.7.2017 & $\begin{array}{l}\text { In einem Bericht des Magazins National Geographic wird die Vermutung geäußert, dass einzelne Geparden } \\
\text { wieder aus Iran nach Turkmenistan eingewandert sein könnten. Die Großkatzenart wurde auf dem Gebiet der } \\
\text { UdSSR bereits vor Jahrzehnten ausgerottet. }\end{array}$ \\
\hline 9.7.2017 & $\begin{array}{l}\text { Präsident Berdymuchammedow kündigt die etappenweise Kürzung staatlicher Mittel für die staatlichen Radio- } \\
\text { und Fernsehsender ab } 2018 \text { bis zu ihrer kompletten Einstellung } 2022 \text { an. }\end{array}$ \\
\hline 10.7.2017 & $\begin{array}{l}\text { Die staatliche Zeitung Turkmenistan - Solotoj Wek (Turkmenistan - Goldenes Jahrhundert) kündigt an, dass } \\
\text { ab dem 1.8.2017 eine Tourismussteuer von } 2 \text { US-Dollar pro Tag von ausländischen Reisenden erhoben wird. }\end{array}$ \\
\hline 10.7.2017 & $\begin{array}{l}\text { In Washington finden US-amerikanisch-turkmenische Regierungskonsultationen mit dem Schwerpunkt auf } \\
\text { Wirtschaftsfragen statt. }\end{array}$ \\
\hline 11.7.2017 & $\begin{array}{l}\text { RFE/RL ruft die turkmenischen Behörden anlässlich des zweiten Jahrestages der Inhaftierung ihres Mitarbei- } \\
\text { ters Saparmamed Nepeskulijew zu dessen Freilassung auf. }\end{array}$ \\
\hline 11.7.2017 & $\begin{array}{l}\text { Außenminister Raschid Meredow trifft in Taschkent mit dem usbekischen Präsidenten Schawkat Mirsijojew } \\
\text { zu Gesprächen über die bilateralen Beziehungen zusammen. }\end{array}$ \\
\hline 14.7.2017 & $\begin{array}{l}\text { OPEC-Generalsekretär Muhammed Barkindo erklärt, dass es mit Turkmenistan keine Einigung über dessen } \\
\text { Anschluss an die Vereinbarung über die globale Begrenzung der Erdölfördermengen gebe. }\end{array}$ \\
\hline 14.7.2017 & $\begin{array}{l}\text { Außenminister Meredow wird in Duschanbe vom tadschikischen Präsidenten Emomali Rachmon zu Gesprä- } \\
\text { chen empfangen. }\end{array}$ \\
\hline 17.7.2017 & $\begin{array}{l}\text { Außenminister Meredow trifft in Tbilisi ein, wo er von seinem georgischen Amtskollegen Michail Dschane- } \\
\text { lidse zu Gesprächen über die bilaterale Zusammenarbeit empfangen wird. }\end{array}$ \\
\hline
\end{tabular}




\begin{tabular}{|l|l|}
\hline 17.7.2017 & $\begin{array}{l}\text { Präsident Berdymuchammedow rügt den Minister für Nationale Sicherheit, Dowrangeldy Bairamow, scharf } \\
\text { für Nachlässigkeiten in seiner Amtsführung. }\end{array}$ \\
\hline 17.7.2017 & In Aschchabad eröffnet mit der Rysgalbank die erste private Bank ihre Hauptfiliale. \\
\hline 17.7.2017 & $\begin{array}{l}\text { Die oppositionelle Website Chronika Turkmenistan meldet, dass Western Union die Maximalsumme von Geld- } \\
\text { überweisungen aus Turkmenistan auf pro Person 1050 Manat (300 US-Dollar) beschränkt habe. (Am 21.7. } \\
\text { widerspricht der Finanzdienstleister dieser Meldung.) }\end{array}$ \\
\hline 18.7.2017 & $\begin{array}{l}\text { Der turkmenische Dienst von RFE/RL meldet, dass mindestens 40 Personen, darunter vor allem ehemalige } \\
\text { Lehrkräfte und Absolventen türkisch-turkmenischer Bildungseinrichtungen, wegen mutmaßlicher Verbindun- } \\
\text { gen zur Gülen-Bewegung zu Haftstrafen zwischen 12 und 20 Jahren verurteilt wurden. Bereits im Januar 2017 } \\
\text { waren 18 Personen aus demselben Grund bis zu 25 Jahre hinter Gitter geschickt worden. }\end{array}$ \\
\hline 18.7.2017 & $\begin{array}{l}\text { Außenminister Meredow trifft zu einem Besuch in Baku ein, wo er u. a. vom aserbaidschanischen Präsidenten } \\
\text { Ilham Alijew zu Gesprächen über das bilaterale Verhältnis empfangen wird und zu Dreiergesprächen mit sei- } \\
\text { nem aserbaidschanischen und türkischen Kollegen zusammentrifft. Nach Angaben des turkmenischen Diens- } \\
\text { tes von RFE/RL unter Berufung auf regierungsnahe Quellen dient Meredows Tournee durch Zentralasien und } \\
\text { den Südkaukasus vor allem dazu, finanzielle Unterstützung für die Asien-Spiele zu akquirieren. }\end{array}$ \\
\hline
\end{tabular}

\section{Usbekistan}

\begin{tabular}{|c|c|}
\hline 24.6 .2017 & Die Zentralbank erhöht als Maßnahme gegen die steigende Inflation den Leitzins vom 9\% auf 14\%. \\
\hline 24.6 .2017 & $\begin{array}{l}\text { Der usbekische Dienst von RFE/RL berichtet unter Berufung auf Angehörige, dass die Türkei am 22.6. heimlich } \\
\text { zwei unter Islamismusverdacht in Istanbul verhaftete usbekische Staatsbürger an Taschkent ausgeliefert habe. }\end{array}$ \\
\hline 25.6.2017 & $\begin{array}{l}\text { In Andischan endet eine weitere Sitzung der Regierungsarbeitsgruppen Usbekistans und Kirgistans zur Deli- } \\
\text { mitierung und Demarkation der gemeinsamen Staatsgrenze. }\end{array}$ \\
\hline 27.6.2017 & $\begin{array}{l}\text { Der ungarische Außenminister Péter Szijjártó wird in Taschkent von seinem Amtskollegen Abdulasis Kami- } \\
\text { low und Premier Abdulla Aripow zu Gesprächen über Stand und Perspektiven der bilateralen Zusammenar- } \\
\text { beit empfangen und eröffnet die Botschaft seines Landes in Usbekistan. }\end{array}$ \\
\hline 27.6.2017 & $\begin{array}{l}\text { Human Rights Watch (HRW) beklagt in seinem neuen, gemeinsam mit dem Uzbek-German Forum for } \\
\text { Human Rights herausgegebenen Bericht über die Situation im Baumwollanbau Usbekistans, dass nach wie } \\
\text { vor Zwangsarbeit verbreitet sei und beschuldigt die Weltbank, Projekte zu unterstützen, bei denen Zwangsar- } \\
\text { beit eingesetzt wird. }\end{array}$ \\
\hline 27.6.2017 & $\begin{array}{l}\text { Eine Sprecherin der Generalstaatsanwaltschaft in Kiew gibt bekannt, dass Akbarali Abdullajew, ein Neffe der } \\
\text { Ehefrau des verstorbenen Präsidenten Islam Karimow, am 14.6. aus dem Hausarrest entlassen und ihm Flücht- } \\
\text { lingsstatus gewährt wurde. Abdullajew wird in Usbekistan wegen des Verdachts von Bestechung und Unter- } \\
\text { schlagung gesucht. Er war im April auf usbekisches Ersuchen bei der Einreise in die Ukraine verhaftet worden. }\end{array}$ \\
\hline 27.6.2017 & $\begin{array}{l}\text { In einer Rede anlässlich des Tages der Mitarbeiter von Presse und Massenmedien fordert Präsident Schaw- } \\
\text { kat Mirsijojew kritische Berichterstattung über problematische Erscheinungen wie Bürokratismus, Korrup- } \\
\text { tion oder Gleichgültigkeit. }\end{array}$ \\
\hline 28.6.2017 & $\begin{array}{l}\text { Bei einem Treffen mit dem Chef der russischen LUKOIL, Wagit Alekperow, in Taschkent äußert sich Präsi- } \\
\text { dent Mirsijojew zufrieden mit der dynamischen Entwicklung der Zusammenarbeit zwischen LUKOIL und } \\
\text { seinem Land im Öl- und Gassektor. }\end{array}$ \\
\hline 28.6.2017 & $\begin{array}{l}\text { Am Rande einer Sitzung des Rates der Innenminister der GUS-Staaten in Duschanbe findet mit einem Gespräch } \\
\text { zwischen Abdusalom Asisow und Ramason Rachimsoda das erste bilaterale Treffen eines usbekischen und tad- } \\
\text { schikischen Innenministers seit } 19 \text { Jahren statt. Gesprächsthemen sind transnationale organisierte Kriminali- } \\
\text { tät, Terrorismus und Extremismus sowie illegaler Drogenhandel. }\end{array}$ \\
\hline 30.6 .2017 & Die Strom- und Gaspreise werden zum 15.7. um ca. $7 \%$ angehoben. \\
\hline 30.6 .2017 & $\begin{array}{l}\text { Präsident Mirsijojew unterzeichnet eine Anordnung, mit der der Öl- und Gassektor Usbekistans umstruktu- } \\
\text { riert wird. }\end{array}$ \\
\hline 1.7.2017 & $\begin{array}{l}\text { Das Textilprotokoll des am 7.4.2011 in Taschkent zwischen EU und Usbekistan unterzeichneten PCA tritt in } \\
\text { Kraft, nachdem es im Dezember } 2016 \text { vom EU-Parlament gebilligt worden war. }\end{array}$ \\
\hline 3.7.2017 & $\begin{array}{l}\text { Auf Vorschlag Präsident Mirsijojews beschließt die bisherige offizielle Jugendbewegung Kamolot (Vollkom- } \\
\text { menheit) auf ihrem 4. Kongress ihre Umbildung in die Union der Jugend Usbekistans. Zum Vorsitzenden wird } \\
\text { Kachramon Kuronbojew gewählt. }\end{array}$ \\
\hline
\end{tabular}




\begin{tabular}{|c|c|}
\hline 3.7.2017 & $\begin{array}{l}\text { RFE/RL meldet, dass der frühere Chef des usbekisch-US-amerikanischen Autokonzerns GM Uzbekistan, } \\
\text { Tochirdschon Dschalilow, der zu Zeiten des ehemaligen Präsidenten Karimow wegen Korruption inhaftiert } \\
\text { war, zum Vorsitzenden des Nahrungsmittelkonzerns Uzbekozikowkatkholding ernannt wurde. }\end{array}$ \\
\hline 4.7.2017 & $\begin{array}{l}\text { In Muinak (AR Karakalpakstan) wird in Anwesenheit des stellvertretenden usbekischen Premier Atschilbaj } \\
\text { Ramatow und des Sprechers des karakalpakischen Parlaments, Musa Erinjasow, eine neue Wasserleitung mit } \\
\text { einer Kapazität von } 7.000 \mathrm{~m}^{3} \text { täglich in Betrieb genommen, mit der nun } 25.000 \text { Menschen mit sauberem Was- } \\
\text { ser versorgt werden können. }\end{array}$ \\
\hline 4.7.2017 & $\begin{array}{l}\text { Die Weltbank gewährt Usbekistan zwei Darlehen in einer Gesamthöhe von knapp } 300 \text { Mio. US-Dollar für } \\
\text { landwirtschaftliche Projekte und ein weiteres in Höhe von knapp } 145 \text { Mio. US-Dollar für die Implementie- } \\
\text { rung der zweiten Phase des Ferghana Valley Water Resources Management Project. }\end{array}$ \\
\hline 5.7.2017 & $\begin{array}{l}\text { Der Präsident der Internationalen Föderation der Gesellschaften des Roten Kreuzes/Roten Halbmonds (IFRC), } \\
\text { Tadateru Konoé, trifft in Taschkent und Samarkand mit der stellvertretenden Premierministerin Tansila Nar- } \\
\text { bajewa u. a. hochrangigen Offiziellen zu Gesprächen zusammen. Es ist dies der erste Besuch eines IFRC-Prä- } \\
\text { sidenten seit Gründung des usbekischen Roten Halbmondes im Jahr } 1925 \text {. }\end{array}$ \\
\hline 5.7.2017 & $\begin{array}{l}\text { Die Redaktion des usbekischen Dienstes der BBC nimmt ihre Arbeit in Usbekistan, die sie } 2005 \text { eingestellt } \\
\text { hatte, wieder auf und sucht lokale Mitarbeiter. }\end{array}$ \\
\hline 5.7.2017 & $\begin{array}{l}\text { Muzaffar Salijew wird von Präsident Mirsijojew zum neuen Minister für Wohnungsbau und kommunale } \\
\text { Dienstleistungen ernannt, nachdem sein Vorgänger Erkin Iskandarow ein anderes Amt übernommen hatte. }\end{array}$ \\
\hline 5.7.2017 & $\begin{array}{l}\text { Eine Anti-Terror-Übung von Sondertruppen des Innenministeriums und des Staatsdienstes für nationale Sicher- } \\
\text { heit auf einem der größten Basare von Taschkent, Uriksor, versetzt die Bevölkerung in Angst und Schrecken. }\end{array}$ \\
\hline 5.7.2017 & $\begin{array}{l}\text { In einer live übertragenen TV-Sendung beantwortet Außenminister Kamilow Fragen von Bürgern, dabei erklärt } \\
\text { er u. a., dass Usbekistan nicht grundsätzlich gegen den Bau des Wasserkraftwerkes Rogun in Tadschikistan sei } \\
\text { und dass man unter bestimmten Bedingungen zur Wiederaufnahme einer Zusammenarbeit mit HRW bereit } \\
\text { sei. Einen Wiedereintritt in die CSTO hält Kamilow dagegen für unwahrscheinlich. }\end{array}$ \\
\hline 6.7.2017 & $\begin{array}{l}\text { In der Freien Wirtschaftszone Dschisak wird der Grundstein für die neuen Produktionsanlagen von SP Uzbe- } \\
\text { kistan Peugeot Citroën Automotive (UzPCA) gelegt. Die Produktion soll } 2019 \text { aufgenommen werden. }\end{array}$ \\
\hline 10.7.2017 & $\begin{array}{l}\text { Premierminister Aripow und der chinesische Landwirtschaftsminister Han Changfu sprechen sich bei einem } \\
\text { Gespräch in Taschkent für eine verstärkte Kooperation beider Staaten im Agrarbereich aus. }\end{array}$ \\
\hline 11.7.2017 & Die Zentralbank setzt den Kurs für 1 US-Dollar auf 4014,5 Som fest, d. i. 24,2\% höher als Anfang 2017. \\
\hline 11.7.2017 & $\begin{array}{l}\text { Präsident Mirsijojew empfängt den turkmenischen Außenminister Raschid Meredow zu Gesprächen über eine } \\
\text { Erweiterung der bilateralen Zusammenarbeit. }\end{array}$ \\
\hline 11.7.2017 & $\begin{array}{l}\text { Die seit } 2006 \text { unterbrochene kürzeste Straßenverbindung zwischen Taschkent und Samarkand über die M-39, } \\
\text { die ca. } 25 \mathrm{~km} \text { durch kasachstanisches Staatsgebiet führt, ist nach Wiederinbetriebnahme der Grenzkontroll- } \\
\text { punkte Malik/Sardarfa und Ok Oltin/Zelinnyj wieder nutzbar. Allerdings berichten usbekische Journalisten } \\
\text { am Ende des ersten Tages von langwierigen Grenzkontrollen auf kasachstanischer Seite. }\end{array}$ \\
\hline 11.7.2017 & $\begin{array}{l}\text { Der Vorsitzende des Komitees für Religionsangelegenheiten, Artukbek Jusupow, und der Vorsitzende des } \\
\text { Muslimrates, Mufti Usmonchon Alimow, werden in Baku vom aserbaidschanischen Präsidenten Ilham Ali- } \\
\text { jew empfangen. }\end{array}$ \\
\hline 11.7.2017 & $\begin{array}{l}\text { Gazeta.uz berichtet, dass aus einer Verordnung von Präsident Mirsijojew über Migrationsprozesse und die Ertei- } \\
\text { lung der Staatsbürgerschaft hervorgehe, dass die Einführung von Auslandspässen beschlossen sei. }\end{array}$ \\
\hline 12.7.2017 & $\begin{array}{l}\text { Eine EU-Delegation unter Führung des Chefs des Generaldirektorats für internationale Zusammenarbeit } \\
\text { und Entwicklung der Europäischen Kommission, Stefano Manservisi, trifft in Taschkent zu Gesprächen mit } \\
\text { Außenminister Kamilow u. a. zur Vorbereitung der 13. Sitzung des Rates EU-Usbekistan ein. }\end{array}$ \\
\hline 13.7.2017 & $\begin{array}{l}\text { In einer Videobotschaft kritisiert Präsident Mirsijojew alle politischen Parteien Usbekistans, weil sie nicht die } \\
\text { ihren Wählern gemachten Versprechungen erfüllen und keine Initiativen zur Realisierung der wichtigsten Auf- } \\
\text { gaben des Landes ergreifen. }\end{array}$ \\
\hline 13.7.2017 & $\begin{array}{l}\text { Bei einem Feuer in einer Fabrikhalle in Kolpino bei Sankt Petersburg kommen sieben usbekische Staatsbür- } \\
\text { ger ums Leben. }\end{array}$ \\
\hline 13.7.2017 & $\begin{array}{l}\text { Außenminister Kamilow empfängt in Taschkent Lilia Burunciuc, die Regionaldirektorin für Zentralasien der } \\
\text { Weltbank, zu Gesprächen über die bilaterale Zusammenarbeit. }\end{array}$ \\
\hline
\end{tabular}




\begin{tabular}{|l|l|}
\hline 17.7.2017 & $\begin{array}{l}\text { Nach Angaben der Nachrichtenagentur UzTAG kamen in Taschkent erstmals seit neun Jahren Vertreter der } \\
\text { Luftfahrtbehörden Kirgistans und Usbekistans zu Gesprächen zusammen. }\end{array}$ \\
\hline 17.7.2017 & $\begin{array}{l}\text { In Brüssel findet die 13. Sitzung des Kooperationsrates Usbekistan-Europäische Union statt. Am Rande des } \\
\text { Treffens unterzeichnen Außenminister Kamilow und Generaldirektor Manservisi eine Vereinbarung über ein } \\
\text { Darlehen in Höhe von 21,5 Mio. Euro für Modernisierung und Verbesserung der Ausstattung von Forschungs- } \\
\text { instituten im Bereich Landwirtschaft und Gartenbau. }\end{array}$ \\
\hline 18.7.2017 & $\begin{array}{l}\text { Außenminister Kamilow trifft in Brüssel mit der EU-Außenbeauftragten Federica Mogherini zu Gesprächen } \\
\text { über Stand und Perspektiven des Verhältnisses Usbekistans zur EU zusammen und erörtert mit dem belgi- } \\
\text { schen Außenminister Didier Reynders Möglichkeiten einer intensivierten Zusammenarbeit in Politik, Han- } \\
\text { del und Kultur. }\end{array}$ \\
\hline 19.7.2017 & $\begin{array}{l}\text { Während einer Pressekonferenz über die Ergebnisse seiner Gespräche mit EU-Vertretern in Brüssel kündigt } \\
\text { Außenminister Kamilow die baldige Schaffung von Bedingungen für die volle Konvertierbarkeit des Sum an. }\end{array}$ \\
\hline 19.7.2017 & $\begin{array}{l}\text { Usbekistan eröffnet in Sankt Petersburg ein Generalkonsulat. Es ist dies nach der Botschaft in Moskau und } \\
\text { dem Generalkonsulat in Nowosibirsk die dritte diplomatische Vertretung Usbekistans in der RF. }\end{array}$ \\
\hline 21.7.2017 & $\begin{array}{l}\text { In Dänemark wird der usbekische Staatsbürger Ibrahimjon Asparow verhaftet, der der Mithilfe für den Anschlag } \\
\text { auf den Nachtclub Reina in Istanbul am 31.12.2016 verdächtigt wird. }\end{array}$ \\
\hline 21.7.2017 & $\begin{array}{l}\text { Seit ihrer Freischaltung vor zehn Monaten wurden 1 Mio. Beschwerden von Bürgern an die virtuelle Bürger- } \\
\text { sprechstunde des Präsidenten gesendet. Die Masse der Zuschriften betraf die Arbeit des Innenministeriums, } \\
\text { teilt das Internetportal der Regierung mit. }\end{array}$ \\
\hline
\end{tabular}

Sie können die gesamte Chronik seit 2008 auch auf <http://www.laender-analysen.de/zentralasien/> unter dem Link »Chronik«lesen.

Herausgeber: Zentrum für Osteuropa- und internationale Studien (Z0iS) gGmbH, Forschungsstelle Osteuropa an der Universität Bremen und Deutsche Gesellschaft für Osteuropakunde e.V.

Die Meinungen, die in den Zentralasien-Analysen geäußert werden, geben ausschließlich die Auffassung der Autoren wieder. Abdruck und sonstige publizistische Nutzung sind nach Rücksprache mit der Redaktion gestattet. Verantwortliche Redakteurin: Dr. Beate Eschment Redaktionsassistenz: Dr. Henryk Alff Satz: Matthias Neumann

Zentralasien-Analysen-Layout: Matthias Neumann, nach einen Konzept von Cengiz Kibaroglu, mit einer Grafik von Sebastian Klüsener Alle Ausgaben der Zentralasien-Analysen sind mit Themen- und Autorenindex archiviert unter www.laender-analysen.de

ISSN 1866-2110 @ 2017 by Zentrum für Osteuropa- und internationale Studien (ZOiS) gGmbH, Forschungsstelle Osteuropa an der Universität Bremen und Deutsche Gesellschaft für Osteuropakunde e.V. Zentrum für Osteuropa- und internationale Studien (ZOiS) $\mathrm{gGmbH} \cdot$ Mohrenstraße 60 • 10117 Berlin • Telefon: + 49 (30) 2005949-20 e-mail: beate.eschment@zois-berlin.de•Internet-Adresse:www.laender-analysen.de/zentralasien 

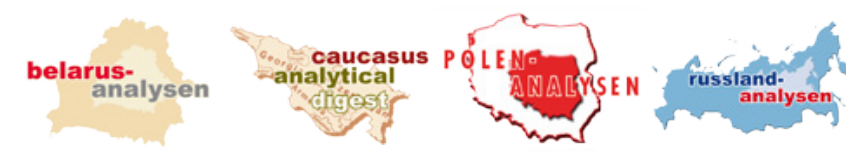

\section{Kostenlose Länder-Analysen auf www.laender-analysen.de}

Die Länder-Analysen bieten regelmäßig im kostenlosen Abonnement kompetente Einschätzungen aktueller politischer, wirtschaftlicher, sozialer und kultureller Entwicklungen in Ostmitteleuropa und der GUS. Sie machen das Wissen, über das die wissenschaftliche Forschung in reichem Maße verfügt, für Politik, Wirtschaft, Medien und die interessierte Öffentlichkeit verfügbar. Autor/innen sind internationale Fachwissenschaftler/innen und Expert/innen. Die Redaktionen der Länder-Analysen bestehen aus Wissenschaftler/innen mit langjähriger Forschungserfahrung.

Die deutschsprachigen Länder-Analysen zur post-sowjetischen Region werden gemeinsam von der Forschungsstelle Osteuropa an der Universität Bremen, dem Zentrum für Osteuropa- und internationale Studien und der Deutschen Gesellschaft für Osteuropakunde herausgegeben. Die Polen-Analysen werden gemeinsam vom Deutschen Polen-Institut, der Forschungsstelle Osteuropa und der Deutschen Gesellschaft für Osteuropakunde herausgegeben. Die englischsprachigen Länder-Analysen erscheinen in Kooperation der Forschungsstelle Osteuropa mit dem Center for Security Studies (CSS) der ETH Zürich.

Die Länder-Analysen bieten regelmäßig Kurzanalysen zu aktuellen Themen, ergänzt um Grafiken und Tabellen sowie Dokumentationen. Zusätzlich gibt es eine Chronik aktueller Ereignisse. Alle Länder-Analysen sind auch mit Archiv und Indizes online verfügbar unter < www.laender-analysen.de $>$.

\section{Belarus-Analysen}

Erscheinungsweise: zweimonatlich

Abonnement unter: <http://www.laender-analysen.de/belarus/>

\section{Caucasus Analytical Digest}

In englischer Sprache. Erscheinungsweise: monatlich

Abonnement unter: <http://www.css.ethz.ch/en/publications/cad.html>

\section{Polen-Analysen}

Erscheinungsweise: zweimal monatlich

Abonnement unter: <http://www.deutsches-polen-institut.de/newsletter/polen-analysen/>

Auch als App für Android ${ }^{\mathrm{TM}}$ (ab Januar 2016) kostenlos auf Google Play ${ }^{\mathrm{TM}}$.

\section{Russland-Analysen}

Erscheinungsweise: zweiwöchentlich

Abonnement unter: <http://www.laender-analysen.de/russland/>

Auch als App für Android (ab Januar 2016) kostenlos auf Google Play.

\section{Russian Analytical Digest}

In englischer Sprache. Erscheinungsweise: zweimal monatlich

Abonnement unter: $<$ http://www.css.ethz.ch/en/publications/rad.html>

\section{Ukraine-Analysen}

Erscheinungsweise: zweimal monatlich

Abonnement unter: <http://www.laender-analysen.de/ukraine/>

Auch als App für Android (ab Januar 2016) kostenlos auf Google Play.

\section{Zentralasien-Analysen}

Erscheinungsweise: monatlich

Abonnement unter: <http://www.laender-analysen.de/zentralasien/>

Auch als App für Android (ab Januar 2016) kostenlos auf Google Play.

\section{Bibliographische Dienste}

Die Bibliographien informieren über englisch- und deutschsprachige wissenschaftliche Neuerscheinungen zu Belarus, Russland, Ukraine sowie zu den zentralasiatischen und kaukasischen Staaten. Erfasst werden jeweils die Themenbereiche Politik, Außenpolitik, Wirtschaft und Soziales.

Erscheinungsweise: viermal jährlich

Abonnement unter: <http://www.laender-analysen.de/bibliographies $>$ 